\title{
Characterization of Geotomographic Studies with the EMRE System
}

\author{
Arto Korpisalo \\ Geological Survey of Finland, P.O. Box 96, 02151 Espoo, Finland \\ Correspondence should be addressed to Arto Korpisalo; arto.korpisalo@gtk.fi
}

Received 13 May 2013; Revised 13 September 2013; Accepted 18 September 2013; Published 20 February 2014

Academic Editor: Andreas Tzanis

Copyright ( 2014 Arto Korpisalo. This is an open access article distributed under the Creative Commons Attribution License, which permits unrestricted use, distribution, and reproduction in any medium, provided the original work is properly cited.

\begin{abstract}
Posiva Oy carries out research and development on spent nuclear fuel disposal in Finland. The repository will be constructed deep in the crystalline bedrock of Olkiluoto island in Eurajoki. Posiva Oy and ANDRA (France) have cooperated actively in examining methods for revealing properties of granitic bedrock. One of the considered methods was an electromagnetic cross-borehole survey, and RIM measurements were conducted in 2009. Olkiluoto migmatitic bedrock has undergone polyphasic ductile-brittle deformation and resistivity in the bedrock varies strongly in range of tens to tens of thousands of $\Omega \mathrm{m}$. Field work was successfully performed in one borehole pair. The results are presented in this paper. The tomographic reconstruction of the borehole section is based on the far-field approximation of the electric field. The results prove that the method can be used between boreholes to delineate and follow sulphide-bearing horizons. The detected low and high resistivity zones and their apparent shapes and orientations are in fair agreement with geological and other geophysical results. The obtained information can be used, for example, in assessing the integrity of the rock mass, as the increased electrical conductivity is often associated with rock mass deformation (clay and water bearing fractures, sulphide and graphite bearing zones).
\end{abstract}

\section{Introduction}

Electromagnetic methods are based on the fact that earth materials may have large contrasts in electrical properties: the electrical conductivity can span several decades ( 10$10^{-5} \mathrm{~S} / \mathrm{m}$ ) in earth, and the relative permittivity can range between 1 and 81 , or even more. The magnetic permeability can be fixed to the value of free space in normal circumstances.

The first studies concerning electromagnetic wave propagation through the earth date back to the beginning of the 20 th century. Sommerfeld and Weyl performed rigorous theoretical studies with vertical antennas, and their formulations are useful even today. Eve and Keys conducted measurements where the propagation of electromagnetic waves through earth materials was also established. The Russian scientist Petrowsky used buried antennas and managed to receive fields in the earth. He calculated the attenuation constants of porphyry and sedimentary rocks at several frequencies [1]. The work was continued by Lager and Lytle [2], Lytle et al. [3], and Somerstein et al. [4]. Stolarczyk and Fry [5] used
RIM to detect faults in the continuity of coal seams, and this can be considered as the starting point of RIM. Russian experts carried out intensive studies using RIM with good results during the late 2000s [6]. They measured the decayed or attenuated field and compared it to the theoretically calculated field decay in a homogeneous medium to estimate the conductivity. The Miningtek Pluto-6 system was developed by the Division of Mining Technology of the CSIR [7]. The frequency synthesis ranges from $1 \mathrm{MHz}$ up to $30 \mathrm{MHz}$, and the gain is effectively adjusted to maintain the power at $1 \mathrm{~W}$. The JW-4 system was developed by the Chinese Institute of Geophysical and Geochemical Exploration (IGGE). The group used a technique where the cross-sectional image was reconstructed from the ratio of decayed fields at two frequencies [8]. In 2010, Geological Survey of Finland (GTK) took into a productive use a RIM system, known as the EMRE system. Today, the application of the RIM technique has been expanded from coal mines, for example, to orebody delineation and mine planning. It is based on the attenuation of electromagnetic signals in the region between two boreholes [9]. The main factor that limits the use of 
radiofrequencies in geophysics is the strong absorption of energy in most earth materials or the penetration being highly frequency dependent. Thus, the expected attenuation rate of electromagnetic waves in a frequency band should be estimated when judging whether the use of RIM is justified in an exploration of the problem.

The measurements can be performed using MOM (multioffset measurement) or ZOM (zero-offset measurement). MOM uses the fan-beam geometry. The idea is to measure a large number of angles passing through the region between the boreholes. In ZOM, both the transmitter and receiver are moved synchronously in the boreholes (parallel-beam geometry). This is a quick and simple technique to locate and define anomalous attenuation zones [10]. According to the ray theory, electromagnetic energy propagates along a straight ray and changes in the electromagnetic field are generated by changes in the material properties along the ray. The straight ray assumption is valid in moderate contrasts when the reconstructed tomographic images can also be reliable [11]. Only the waves that propagate in the first Fresnel zone (e.g., direct and multipath waves) are generally strong enough and detectable in the EMRE system. In addition, the smallest structure that can be resolved can be estimated by the width of the first Fresnel zone. The performance level of the EMRE system is estimated using a simplified model [12].

When the amplitude data are collected, the attenuation can be estimated directly [11]. Under certain conditions it might also be possible to convert the low Q-domain attenuation to the electric conductivity and use it to determine the relative permittivity in the high Q-domain [13]. Using a plane wave assumption, which is valid in the far field, the measured amplitudes can be converted to the attenuation distribution in a simple way [14]. Olkiluoto served as a testing site for the RIM measurements to determine the capacity and usefulness of RIM in determining the structural integrity of the rock in the area Figure 7. Despite a challenging measurement geometry and the sulphide-rich areas in the bedrock, the measurements and results were successful and good. However, the smaller-scale fractures could not be distinguished in the conductive rock mass.

The objective of this paper is to demonstrate the usability of a continuous wave device (CW) [15] in different electrical conditions and to clarify RIM's transillumination properties as a function of electrical conductivity, relative permittivity, magnetic permeability, and frequency. In addition, a special field case is illustrated. According to the results, the RIM technique can be usefully applied in various electrical environments. Figure 1 presents the main components of the EMRE system.

\section{Theory}

A cross-borehole EM survey has several benefits over ground-level electromagnetic sounding methods. Applying a borehole transmitter brings the survey closer to the target and will allow the use of higher frequencies, thus enabling a higher spatial resolution. Another benefit is the possibility to view the target from different angles and directions, not only in the vertical direction from the earth's surface. Having the transmitter in a borehole eliminates the boundary effects related to the ground surface and the strong attenuation emerging from soil deposits. A drawback is the suboptimal availability and location of boreholes and limited power of transmission of borehole probes. The physical behaviour of an electromagnetic field is governed mathematically by Maxwell equations, which describe the relationship between electric and magnetic fields in a medium and quantify the material properties [16-18].

In the EMRE system, the electromagnetic fields are generated by insulated electric dipole antennas [19] that are aligned parallel to the borehole axis (Figure 1). The electromagnetic field of the electric dipole has three effective components: a magnetic component $\left(B_{\phi}\right)$, an electric tangential component $\left(E_{\Theta}\right)$, and a radial component $\left(E_{r}\right)$. The electric field determines the polarization, and the maximal coupling with targets is achieved when the field component is parallel to the target.

The field depends on the distance $\bar{r}$ from the source. Certain characteristics of an electromagnetic field dominate at one particular distance from the antenna, while a completely different behaviour can dominate at another location. The wave number $\bar{k}$ multiplied by the distance $\bar{r}$ defines the behaviour $[10,11,13]$. When $|\bar{k} \cdot \bar{r}| \ll 1$ or at a distance much shorter than the wavelength, the electric field resembles the static dipole field, being proportional to $r^{-3}$ (static term), and anything in this region that couples with the antenna will disturb the antenna severely (reactive near field). The EMRE system operates in the near-field domain due to the wavelengths of hundreds of metres when low frequencies $(f<300 \mathrm{kHz})$ and short distances $(r<50 \mathrm{~m})$ are used in a resistivity range of $<1000 \Omega \mathrm{m}$. When $|\bar{k} \cdot \bar{r}|=1$, the field is proportional to $r^{-2}$ (induction term), and the static term $\left(\sim r^{-3}\right)$ becomes negligible. The region is known as a radiating near-field or Fresnel zone. In the frequency range of $300-2500 \mathrm{kHz}$ and with resistivities $<10000 \Omega \mathrm{m}$, the EMRE system operates in the radiating domain. When $|\bar{k} \cdot \bar{r}| \gg 1$ or at a distance much greater than the wavelength, the electric field is inversely dependent on the distance $r^{-3}$ (radiation term). The angular field distribution is independent of the distance from the antenna (far-field or Fraunhofer zone). When frequencies $f>1000 \mathrm{kHz}$ and distances $r>100 \mathrm{~m}$ are used in the resistivity range of $>10000 \Omega \mathrm{m}$, the EMRE system is operating in the far field. The inhomogeneous generalized time-domain wave equations for the time harmonic electric field $E$ and magnetic field $H$ ( $e^{i \omega t}$ dependence) can be written as $[16-18]$

$$
\begin{gathered}
\nabla^{2} \bar{E}-\mu \sigma \frac{\partial \bar{E}}{d t}-\mu \varepsilon \frac{\partial^{2} \bar{E}}{\partial t^{2}}=\frac{1}{\varepsilon} \nabla \rho+\mu \frac{\partial \bar{J}}{\partial t}, \\
\nabla^{2} \bar{H}-\mu \sigma \frac{\partial \bar{H}}{d t}-\mu \varepsilon \frac{\partial^{2} \bar{H}}{\partial t^{2}}=-\nabla \times \bar{J} .
\end{gathered}
$$

On the right-hand side are the sources of the fields. On the left-hand side, the second-order time-derivative term is the wave term (oscillating term) with an energy storage factor $(\mu \varepsilon)$, and the first-order time-derivative term is the damping 


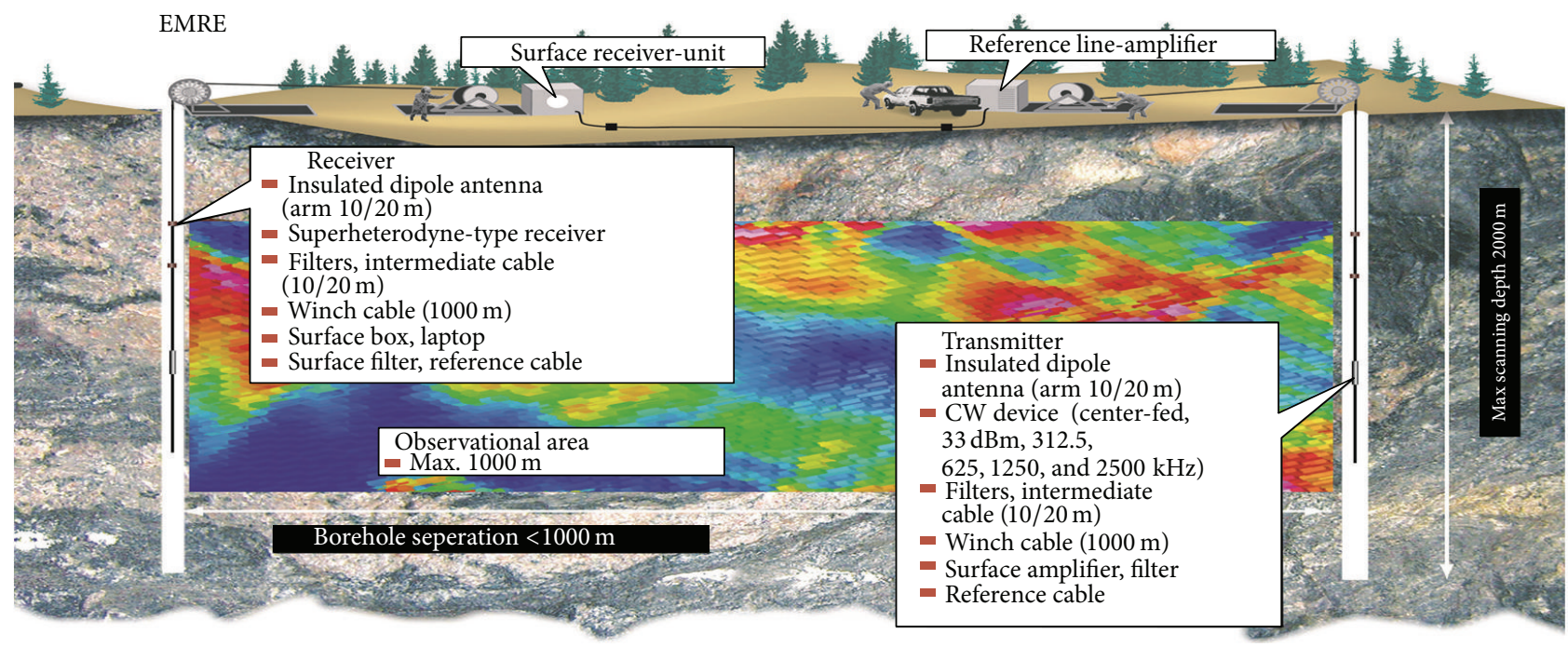

FiguRE 1: The EMRE device and measurement geometry (not to scale). The mobile receiver takes measurements during movement at a frequency of $2 \mathrm{~Hz}(\Delta \sim 0.25 \mathrm{~m}, \sim 30 \mathrm{~m} / \mathrm{min})$, while the stable transmitter transmits continuously $(312.5,625,1250,2500 \mathrm{kHz})$. The attenuation distribution of the borehole section is reconstructed $(\mathrm{dB} / \mathrm{m})$, a red colour corresponding to higher attenuation.

term with an energy dissipation factor $(\mu \sigma)$. For sinusoidal time-harmonic fields $\left(\sim e^{i \omega t}\right)$, these equations $(\rho=0$ and $J=$ 0 ) become frequency-domain Helmholtz's equations by using the following substitutions: $\partial / \partial t \rightarrow i \omega, \partial^{2} / \partial t^{2} \rightarrow-\omega^{2}$,

$$
\begin{aligned}
\nabla^{2} \bar{E}-i \omega \mu \sigma \bar{E}+\omega^{2} \mu \varepsilon \bar{E} & =0 \longrightarrow \nabla^{2} \bar{E}+k^{2} \bar{E}=0 \\
\nabla^{2} \bar{H}-i \omega \mu \sigma \bar{H}+\omega^{2} \mu \varepsilon \bar{H} & =0 \longrightarrow \nabla^{2} \bar{E}+k^{2} \bar{E}=0
\end{aligned}
$$

where $k^{2}=\omega^{2} \mu \varepsilon-i \omega \mu \sigma$ is the complex wave number [16-18].

Depending on the magnitude of energy loss (conductivity) relative to energy storage (permittivity, permeability), the fields may diffuse or propagate as waves. The $Q$-factor (inverse loss tangent) describes the behaviour of the field [10, 20]:

$$
Q=\frac{\varepsilon^{\prime}-\sigma^{\prime \prime} / \omega}{\sigma^{\prime}+\omega \varepsilon^{\prime \prime}}=\frac{\omega \varepsilon^{\prime}-\sigma^{\prime \prime}}{\sigma^{\prime}+\omega \varepsilon^{\prime \prime}}=\tan \Phi^{-1} \approx \frac{\omega \varepsilon^{\prime}}{\sigma^{\prime}} .
$$

In its effective form (4), the Q-factor contains the imaginary components of complex conductivity $\sigma^{\prime \prime}\left(\sigma=\sigma^{\prime}+i \sigma^{\prime \prime}\right)$ and permittivity $\varepsilon^{\prime \prime}\left(\varepsilon=\varepsilon^{\prime}+i \varepsilon^{\prime \prime}\right)$. The loss tangent of any material $(\tan \phi)$ quantitatively describes dissipation of the electric energy due to different physical processes such as electrical conduction, dielectric relaxation and resonance, and loss from nonlinear processes (hysteresis).

When $Q \gg 1$ (high frequency/low conductivity), the dielectric properties are dominant $\left(\omega \varepsilon^{\prime} \gg \sigma^{\prime}\right)$, and the electromagnetic fields propagate as waves through the medium. All the frequency components travel at roughly the same velocity (nondispersive) and encounter the same negligible attenuation $\alpha$ and the waveform remains unaltered through the passage. Reflection, scattering, refraction, and diffraction effects on the boundaries are possible due to the different electrical properties, resulting, for instance, in multipath waves. The velocity $v$, attenuation rate $\alpha$, phase shift $\beta$, wave impedance $\eta$, and wave number $k$ can be written as

$$
\begin{gathered}
\nu=\frac{1}{\sqrt{\varepsilon \mu}}, \quad \eta=\sqrt{\frac{\mu}{\varepsilon}} \approx 377 \Omega, \\
\alpha=\sqrt{\frac{\mu}{\varepsilon}} \cdot \frac{\sigma}{2}, \quad \beta=\omega \sqrt{\mu \varepsilon}, \quad k^{2}=\omega^{2} \mu \varepsilon .
\end{gathered}
$$

The attenuation rate is dependent on the dielectric permittivity $\varepsilon$, magnetic permeability $\mu$, and electrical conductivity $\sigma$ but not on the frequency $\omega$ (nonfrequency dependent media, but for rock, it is frequency dependent). The phase shift is dependent on the frequency, permittivity, and permeability. When $\sigma=0$, the wave impedance has the value of a vacuum or $\eta \approx 377 \Omega$. The wave impedance is an important factor when reflection and transmission coefficients are concerned in the boundaries [17].

When $Q \ll 1\left(\omega \varepsilon^{\prime} \ll \sigma^{\prime}\right)$, the velocity $v$ is independent of the dielectric permittivity $\varepsilon$ (quasi-static) but is controlled by the product of conductivity $\sigma$ and permeability $\mu$. The energy distribution is dispersive; that is, the attenuation $\alpha$ will depend on the conductivity $\sigma$, permeability $\mu$, and frequency $\omega$ :

$$
\begin{gathered}
v=\sqrt{\frac{2 \omega}{\mu \sigma}}, \quad \eta=\sqrt{\frac{i \omega \mu}{\sigma}}=\sqrt{\frac{\omega \mu}{2 \sigma}}(1+i)=\sqrt{\frac{\omega \mu}{\sigma}} e^{i(\pi / 4)}, \\
k^{2}=-i \omega \mu \sigma, \quad \alpha=\sqrt{\frac{\omega \mu \sigma}{2}}=\frac{1}{\delta}=\beta
\end{gathered}
$$

Thus, the phase shift is equal to the attenuation constant (7). One wavelength $\lambda$ means a phase shift of $2 \pi$ radians. It is also the distance corresponding to $2 \pi$ Nepers $(\sim 55 \mathrm{~dB})$ of attenuation $(1 \mathrm{Neper}=8.686 \mathrm{~dB})$. In addition, one skin 
depth, a distance where the field has attenuated to $e^{-1}$ of the initial field strength, means an attenuation of 1 Neper $(\sim 9 \mathrm{~dB})$, corresponding to one radian length $\left(\sim 57^{\circ}\right)$. In the low Q-domain, the attenuation is equal to the phase shift and can be described in terms of skin depth $\delta(7)$. The skin depth is a plane wave factor that is attached to damping fields. The induced volume currents can be replaced by the surface currents and the current is concentrated on the surface of the target. The approximation holds both in the high frequency domain, when the conductivity has to be large enough, and in the low frequency domain, when the target can be more resistive. In conductors, the wave impedance is complex valued. Thus, the electric and magnetic fields are no longer in phase (6). For almost all rocks, the magnetic susceptibility is $\chi_{\mathrm{m}} \ll 1\left(\mu_{r}=1+\chi_{\mathrm{m}}\right)$, and the permeability is thus $\mu \cong \mu_{0}$ or $\mu_{r}=1$. In the wave equations (1), the permeability $\mu$ appears as a product with conductivity $\sigma(\mu \sigma)$. Due to the large range of conductivity variation in the Earth's materials $\left(\sim 10-10^{-5} \mathrm{~S} / \mathrm{m}\right)$, the conductivity outweighs the permeability changes $\left(\mu_{r} \approx 1\right)$. However, it is evident that the effect of increasing the permeability is to increase the attenuation and phase shift. In quasi-static conditions (diffusive domain), electromagnetic fields do not experience the above-mentioned effects on the boundaries (e.g., reflection, refractions), but the fields diffuse over the boundaries like heat [17].

When $Q \approx 1\left(\omega \varepsilon^{\prime} \cong \sigma^{\prime} \leftrightarrow f \approx \sigma^{\prime} / 2 \pi \varepsilon^{\prime}\right)$, the system is in the intermediate domain where the electric permittivity and conductivity and magnetic permeability must be taken into account when considering attenuation rates and phase constants (6). To estimate the phase shift $(\beta)$ and attenuation rate $(\alpha)$ in a dissipative medium, more general expressions must be used for the phase and attenuation constants:

$$
\begin{aligned}
\beta & =\omega \sqrt{\frac{\mu \varepsilon}{2}}\left(\sqrt{1+\left(\frac{\sigma}{\omega \varepsilon}\right)^{2}}+1\right)^{1 / 2} \\
& =\omega \sqrt{\frac{\mu \varepsilon}{2}}\left(\sqrt{1+\tan ^{2} \Phi}+1\right)^{1 / 2}(\mathrm{rad} / \mathrm{m}) \\
\alpha & =8.686 \cdot \omega \sqrt{\frac{\mu \varepsilon}{2}}\left(\sqrt{1+\left(\frac{\sigma}{\omega \varepsilon}\right)^{2}}-1\right)^{1 / 2} \\
& =8.686 \cdot \omega \sqrt{\frac{\mu \varepsilon}{2}}\left(\sqrt{1+\tan ^{2} \Phi}-1\right)^{1 / 2} \quad(\mathrm{~dB} / \mathrm{m})
\end{aligned}
$$

The phase velocity $v$, the wavelength $\lambda$, and wave number $k$ for a dissipative medium are obtained using the following general formulas [17]:

$$
\begin{aligned}
v_{p} & =\frac{\omega}{\beta}=\frac{1}{\omega \sqrt{2 / \mu \varepsilon}\left(\sqrt{1+(\sigma / \omega \varepsilon)^{2}}+1\right)^{1 / 2}} \\
& =\frac{1}{\omega \sqrt{2 / \mu \varepsilon}\left(\sqrt{1+\tan ^{2} \Phi}+1\right)^{1 / 2}},
\end{aligned}
$$

$$
\begin{gathered}
\lambda=\frac{2 \pi}{\beta}=\frac{1}{f} \sqrt{\frac{2}{\mu \varepsilon}}\left(\sqrt{1+\left(\frac{\sigma}{\omega \varepsilon}\right)^{2}}+1\right)^{-1 / 2} \\
=\frac{1}{f} \sqrt{\frac{2}{\mu \varepsilon}}\left(\sqrt{1+\tan ^{2} \Phi}+1\right)^{-1 / 2}, \\
k^{2}=\omega^{2} \mu \varepsilon-i \omega \mu \sigma .
\end{gathered}
$$

Figures 2(a)-2(b) present the cut-off frequencies $(f=$ $\left.\sigma^{\prime} / 2 \pi \omega\right)$ for different materials $(\rho=100,1000,10000 \Omega \mathrm{m}$ and $\left.\varepsilon_{r}=10,20\right)$.

In a low resistive medium $\left(\rho=100 \Omega \mathrm{m}, \varepsilon_{r}=10\right)$, the quasi-static conditions (displacement current negligible) are valid up to $\sim 1000 \mathrm{kHz}$, while, in a highly resistive environment ( $\rho=10000 \Omega \mathrm{m}$ ), the quasi-static conditions are valid up to $\sim 10 \mathrm{kHz}$ (Figure 2(a)). Increasing the relative permittivity of the medium, the cut-off frequencies decrease $\left(\varepsilon_{r}=20\right)$ and the quasi-static conditions are valid almost up to $\sim 60 \mathrm{kHz}$, while, in a highly resistive environment ( $\rho=$ $10000 \Omega \mathrm{m}$ ), the quasi-static conditions are valid up to $\sim 5 \mathrm{kHz}$ (Figure 2(b)).

The main factor that limits the use of radiofrequencies in geophysics is the strong absorption of energy in most earth materials or the penetration being highly frequency dependent. Thus, the expected attenuation rate of electromagnetic waves in a frequency band should be estimated when considering whether the use of RIM is justified in the research area. The behaviour of the attenuation rates as a function of the frequency and the electrical properties could be investigated using (8). The relative permittivity and permeability have minor effects on the attenuation rates in the EMRE band $(312.5-2500 \mathrm{kHz})$, but the electric conductivity is the main property that effectively controls the transillumination ranges. Figure 3 presents the log-log plots of attenuation rates as a function of conductivity for various relative permittivity values. The frequency and relative permeability are fixed. At lower conductivities, the respective permittivity curves are parallel and the attenuation rate is lower at higher values of permittivity.

In resistive materials, the attenuation is dependent on the permittivity value, but at the critical conductivity values of $10^{-2}-10^{-1} \mathrm{~S} / \mathrm{m}$ the curves merge together to become identical, and this happens sooner at lower frequencies. This is clear because (8) reduces to a form of $\alpha \cong \sqrt{\sigma \mu \omega}$ when the loss tangent is much greater than unity. The attenuation rates increase as the conductivity increases in all plots (Figure 3 ).

The attenuation rates of rocks can also be measured directly as in situ measurements. Direct measurement, in which the reduction in the amplitude of an electromagnetic wave due to a known distance of material is measured, is the most accurate method of measuring the attenuation rate. Vogt [7] reported a capacitive method in which small cylindrical pieces of different rock types were carefully prepared and placed as a dielectric in the capacitor. Thus, measuring the complex impedance $Z$ of the capacitor, the electrical conductivity $\sigma$ and the relative permittivity $\varepsilon_{r}$ of the sample could be calculated. The reliability of the results is 


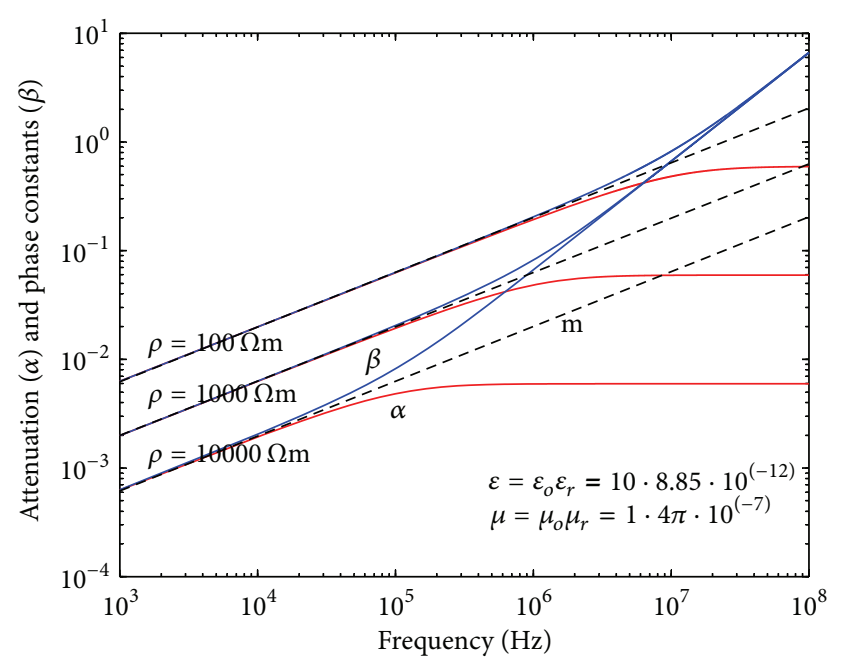

(a)

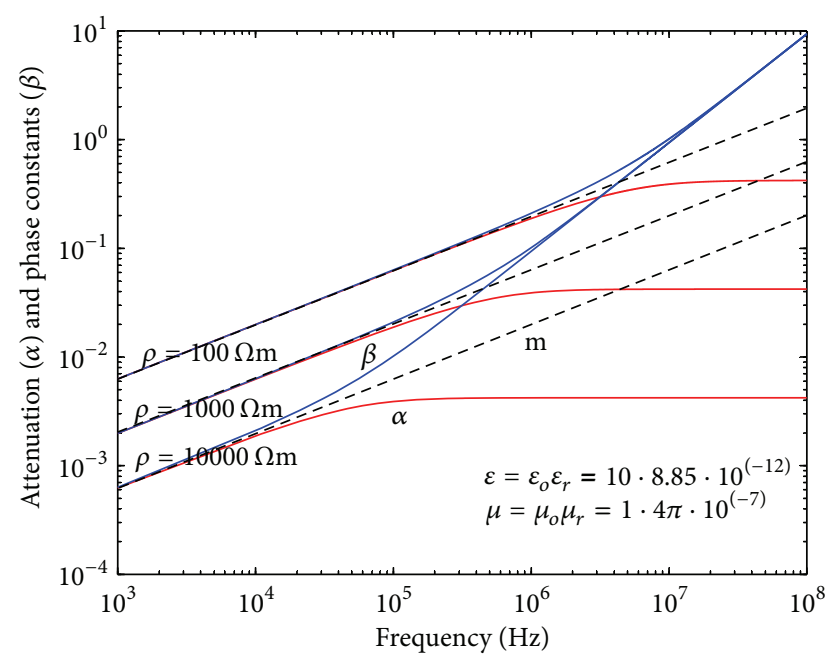

(b)

Figure 2: (a) The attenuation $(\alpha)$ and phase $(\beta)$ constants in a dissipative medium with $\varepsilon_{r}=10$ and $\rho=100,1000$, and 10000 $\Omega$ m. (b) The phase and attenuation constants in a dissipative medium with $\varepsilon_{r}=20 . \alpha$ is the attenuation (red lines) as Nepers/m and $\beta$ is the phase constant (blue lines) as $\mathrm{rad} / \mathrm{m}$. The $\mathrm{m}$-curve is valid when the attenuation constant equals the phase constant or $\alpha \approx \beta$ (black curves).

not good, because the electrical properties are highly variable, for example, due to moisture changes in the samples and a scale dependence in which different factors are involved [7]. On the other hand, small-scale variations may thus be lost in situ measurements. When the measured conductivities and permittivities are concerned, the conductivity must be introduced in its effective form $\sigma_{\text {eff }}=\sigma^{\prime}-\omega \varepsilon^{\prime \prime}$ and the permittivity in a form of $\varepsilon_{\text {eff }}=\varepsilon^{\prime}+\sigma^{\prime \prime} / \omega$, where $\sigma^{\prime \prime}$ and $\varepsilon^{\prime \prime}$ are the imaginary parts of complex conductivity (dielectric conductivity) and permittivity (conductive permittivity). The dielectric conductivity $\sigma^{\prime \prime}$ takes account of time lags in conduction and the conductive permittivity $\varepsilon^{\prime \prime}$ takes account of time lags in polarization. Vogt estimated the properties of rock (conductivity and permittivity), fitting the measured resistances $R$ and reactances $X(Z=R+i X)$ to Debye models. The Debye model results for two samples (granite and sulphide sample) together with the derived properties (attenuation, wavelength, velocity, and loss tangent) are plotted as black curves in Figure 4 [7].

Figure 4 presents the results of capacitive measurements of two small cylindrical rock samples to determine the electrical conductivity and relative permittivity and the derived properties. The measurements include a certain degree of equipment-based inaccuracy, and error bars are therefore included in the plots. The error bars are a result of the accuracy specification of the instrument. In highly resistive media the attenuation estimate is not good. In conductive media, the amplitude measurement is unreliable, being too low, and the relative permittivity is thus obscure [7]. Granite is a highly resistive rock type in which the effective permittivity remains almost at a constant value as a function of frequency, perhaps having a slightly decreasing trend, meaning that the dielectric conductivity $\sigma^{\prime \prime}$ has a minor effect on the permittivity. The effective conductivity is dominated by the real component at low frequencies, while at higher frequencies the dielectric conductivity component $\varepsilon^{\prime \prime}$ starts to dominate and has an increasing effect on the conductivity. The infected attenuation rate is low, being $\sim 0.02 \mathrm{~dB} / \mathrm{m}(\sim 1 \mathrm{MHz})$; thus, the transillumination distances should be large (Figure 4(a)). Sulphide is a good conductor. The effective conductivity is almost constant (DC value), and the real component is therefore dominant and the dielectric conductivity component $\varepsilon^{\prime \prime}$ is negligible. The permittivity has a strongly decreasing trend and is strongly inflected by the inaccuracy. The attenuation rates increase strongly as a function of frequency. The infected loss tangents are high, meaning high attenuation rates that reduce translumination distances [7] (Figure 4(b)).

The resistivity of Olkiluoto migmatitic bedrock varies strongly in the range of tens to tens of thousands of $\Omega \mathrm{m}$ due to highly conductive disseminated sulphide bearings. The theoretical attenuation rates (Figure 3 ) and the results from the capacitive measurements (Figure 4) by Vogt [7] are in good agreement with highly resistive granite: $\alpha \ll 0.1 \mathrm{~dB} / \mathrm{m}$, $\rho<10000 \Omega \mathrm{m}$ in the whole EMRE band. Sulphide is a good conductor and the electrical contrast with the host rock is considerable. However, we have great confidence in the method and that it can reliably be used to delineate and follow sulphide-bearing horizons in the area. The theoretical estimates of the performance level of the EMRE device (Table 2) also strongly support these opinions.

\section{Rim Instrument}

In Finland, the radiofrequency imaging method (RIM) has been used since the pioneering work by Korpisalo et al. in 2005 [21-23]. The effective performance of any radiating system depends on the characteristics of transmitter and receiver antennas, because they are the important intermediate devices that radiate and capture the propagating electromagnetic energy [19]. The core of the EMRE system 


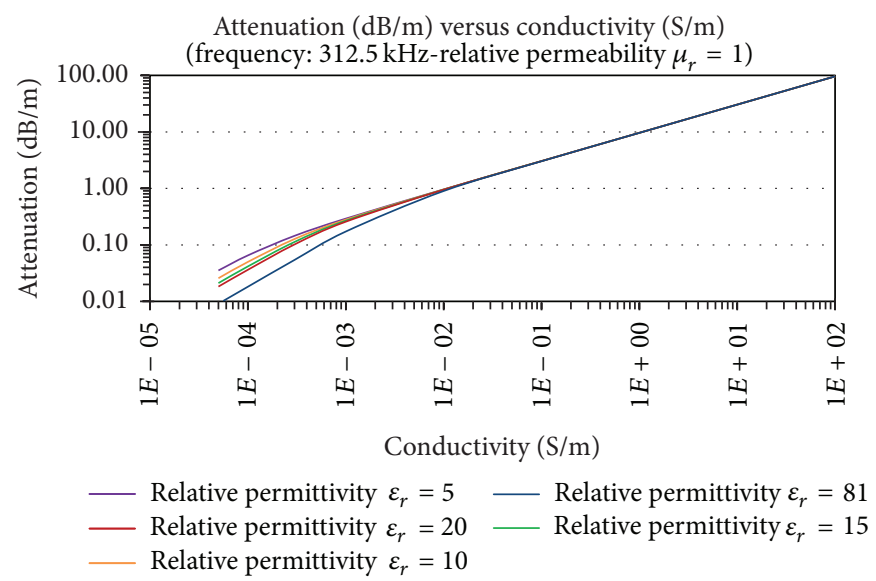

(a)

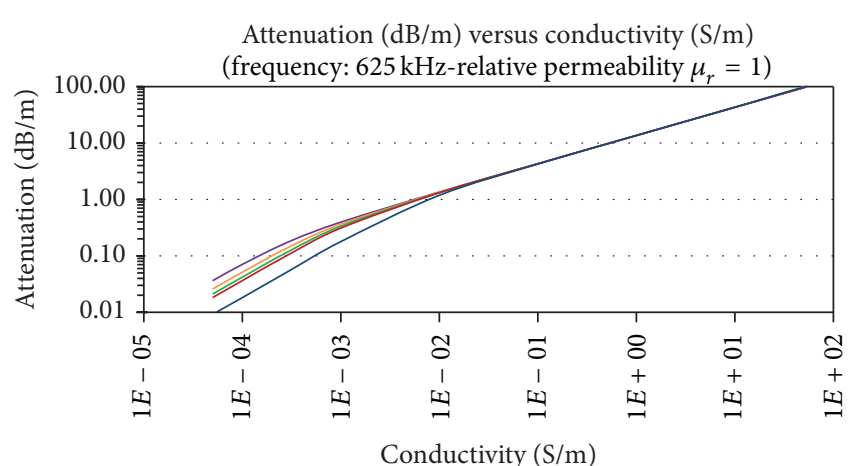

$\begin{array}{ll}\text { Relative permittivity } \varepsilon_{r}=5 & - \text { Relative permittivity } \varepsilon_{r}=81 \\ \text { Relative permittivity } \varepsilon_{r}=20 & \text { Relative permittivity } \varepsilon_{r}=15 \\ \text { Relative permittivity } \varepsilon_{r}=10 & \end{array}$

(b)

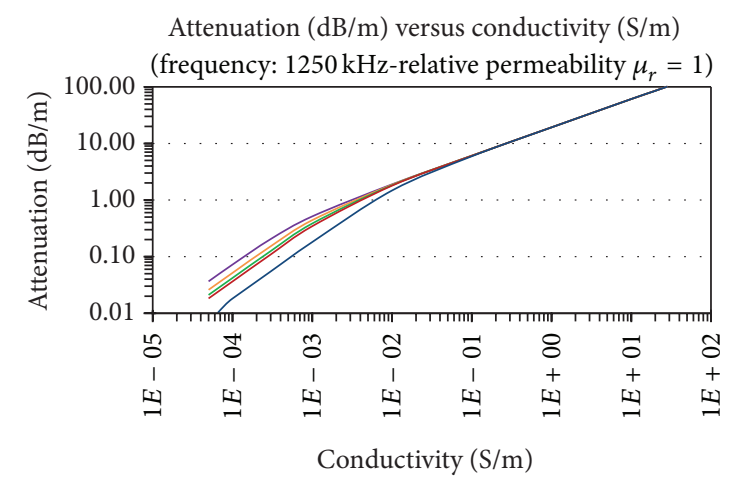

Relative permittivity $\varepsilon_{r}=5$

Relative permittivity $\varepsilon_{r}=20$

Relative permittivity $\varepsilon_{r}=10$

(c)

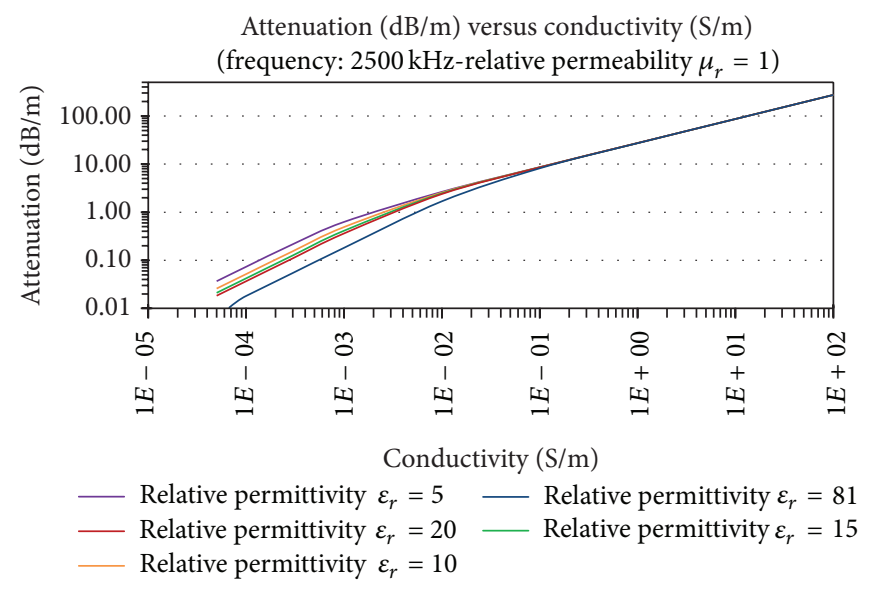

(d)

FIGURE 3: The attenuation presented as a function of conductivity in various materials. The relative permittivity ranges between $\varepsilon_{r}=5$ and $\varepsilon_{r}=81$ (water). The relative permeability is fixed at the free space value (1). (a) Frequency $312.5 \mathrm{kHz}$, (b) frequency $625 \mathrm{kHz}$, (c) frequency $1250 \mathrm{kHz}$, and (d) frequency $2500 \mathrm{kHz}$. 

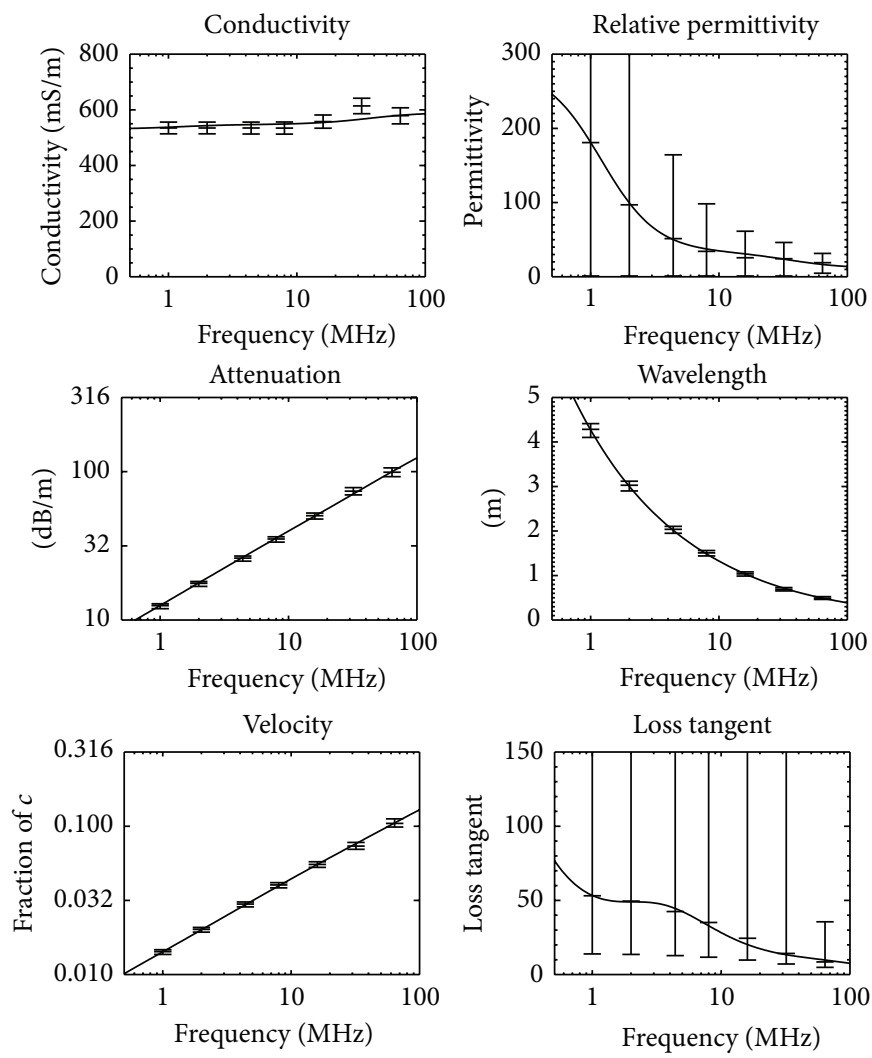

(a)
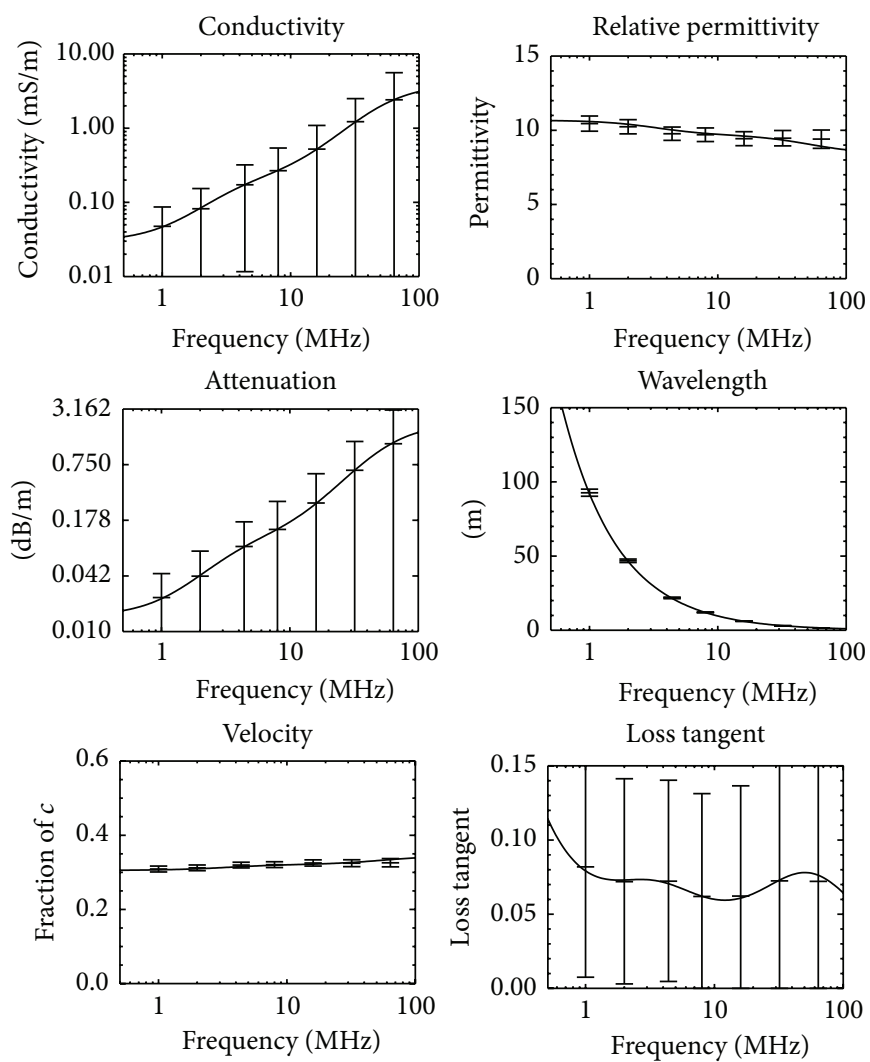

(b)

Figure 4: The electrical properties of a granite sample (a) and a sulphide sample (b) from Vogt [7]. Error bars are included and they are a result of the accuracy specification of the device. 
is a Russian-based device [24] that simultaneously operates at a maximum of four frequencies: $312.5,625,1250$, and $2500 \mathrm{kHz}$ (Figure 5(a)) [25]. Thus, the survey time is reduced by measuring all frequencies simultaneously. The amplitude of the tangential component of the electric field (far-field approximation) and the relative phase difference are measured along the axis of the receiver borehole. The reference signal $(156.25 \mathrm{kHz})$ generated in the borehole transmitter is essential in a tuning operation of the receiver when the receiver is locked to the reference frequency (Figure 5(b)). Without a reference signal, the detection of phase difference would be impossible, but even if the tuning is not functioning properly, the equipment can be used to detect the amplitudes (Figure 5(c)). The measurements are controlled and information is recorded by a laptop. The automated measurement process requires minimal intervention by the operator. The operator can monitor the data quality from the laptop's screen at all frequencies in real time and view previous results. This allows quick adjustments to the survey depth range and makes the shortening or lengthening of the transmitter step size easy and will enable decisions on rapid remeasurement.

3.1. Transmitter. The borehole transmitter consists of a halfway dipole antenna $20 / 40 \mathrm{~m}$ in length, an intermediate cable $10 / 20 \mathrm{~m}$ in length, and a control tube for both the corresponding electronics and voltage source (12 accumulators, $3500 \mathrm{mAh}$ ), that is, $1.5 \mathrm{~m}$ in length. It is a CW device [15]. Low-pass filters are used to galvanically isolate the antenna from the winch cable (Figure 1).

Figure 5(b) presents a block diagram of a CW device. The oscillator (e.g., quartz crystal) generates the radiofrequency (RF) carrier at the basic frequency of $2500 \mathrm{kHz}$, maintaining it accurately. The other measurement frequencies $(1250,625$, and $312.5 \mathrm{kHz})$ and the vital reference frequency $(156.25 \mathrm{kHz})$ are generated from a basic frequency in a frequency divider circuit (F/2-4-8-16). Measurement frequencies can be freely selected via the jumper settings $(S)$. The selected frequencies are conducted to the power amplifiers $(E)$ through the buffer amplifiers $(B)$. Applying a transformer circuit $(T)$, the RF signals are sent to the antenna. Thus, the antenna's function is to serve as an interface between the generator and the surrounding environment. The nominal output power of the transmitter is $2 \mathrm{~W} / 33 \mathrm{dBm}$. Normally, the power is shared out between the four frequencies. However, a frequency can be rejected from the normal operation in a special situation to channel the whole power to the remaining frequencies. The reference frequency of $156.25 \mathrm{kHz}$ is sent through the winch cable to the Earth's surface. After the amplification, it is channelled through the ground-level reference cable to the receiver box, where it is used in the tuning operation. When receiver tuning is accomplished, the receiver is locked to the reference frequency, making phase detection possible. The reference frequency is low enough not to disturb the measurements [15].

3.2. Receiver. The borehole receiver has the same half-way antennas and cables as the transmitter, but the control tube for the electronics and voltage source (12 accumulators,
$3500 \mathrm{mAh}$ ) is $2.5 \mathrm{~m}$ long. The galvanic isolation is performed using the same type of low-pass filters as in the transmitter. The receiver is established in a manner that does not deviate from a normal radio. The front end of the receiver (RF amplifier) and the mixers are situated in the borehole unit. The normal components (e.g., detector, $\mathrm{AD}$ transformation) are situated in the surface box. Technically, it is a normal superheterodyne-type receiver in which a long winch cable is used to transmit the modulated signals up to the detector [15].

Figure 5(c) presents a diagram of the receiver. The incoming radiofrequency $(\mathrm{RF})$ signals are applied to the band-pass filter and RF amplifier. The RF amplifier improves the signalto-noise ratio and provides sufficient selectivity. It determines the sensitivity of the receiver. This stage is also known as a preselector stage (the front end of the receiver). The RF amplifier and a local oscillator are connected in a circuit called a mixer $(M)$. The signals from the preselector and the signal from a local oscillator $(2600 \mathrm{kHz})$ are heterodyned, producing, for example, the four constant difference frequencies (100-50-25-12.5 kHz) and their harmonics. The constant frequencies of the converted signal are known as the intermediate frequencies (IF frequencies). The mixer stage is also known as a first detector stage. The data are transferred from the borehole to the surface using a pair cable to which two filters are connected. They are used to isolate the antennas galvanically from the winch cables and to function as the low-pass filters. The first IF band $(100-12.5 \mathrm{kHz})$ is several octaves, and another mixing stage is performed where the first IF frequency band is modulated with a carrier frequency of $162.50 \mathrm{kHz}$ that is generated from the basic frequency of the receiver crystal $(2600 \mathrm{kHz})$. The new band is less than one octave, and the data transfer is not so challenging. The receiver is tuned or locked to the reference frequency $(156.25 \mathrm{kHz})$ using the tracking option of the receiver to keep the modulation results in the limits of band-pass filters (B), where the signals are passed to the detection stages after demodulation, one for each frequency, providing reliable production of the relative phases and amplitudes. Thus, the first detection stage is carried out in the borehole unit, and the winch cable is used as a guiding path for the modulated signals to the surface where the final detection is performed after the demodulation [15].

The receiver has antennas similar to the transmitter. It is not compulsory to use antennas of the same length in transmission and reception. It is usual to use a longer antenna in transmission to make use of the best possible efficiency and at the same time to use a shorter antenna in reception to maintain the best possible resolution. The receiver has the same impedance mismatching problem as the transmitter, and the load (antenna) does not absorb all the RF power that reaches it. Instead, some of the RF power is sent back towards the signal source when the signal reaches the point where the line is connected to the load. This is known as reflected power or reverse power. The receiver unit consists of an internal calibration unit where the constant valued fields of 100, 10, and $1 \mu \mathrm{V}$ can be fed to the antenna input of the receiver. Thus, the measured amplitude values can be converted to the actual $\mu \mathrm{V}$ units. The receiver has a dynamic range of $\sim 40 \mathrm{~dB}$, which 


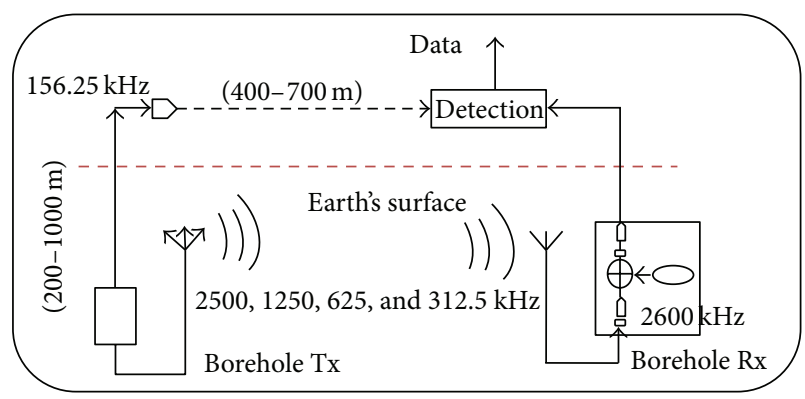

(a)

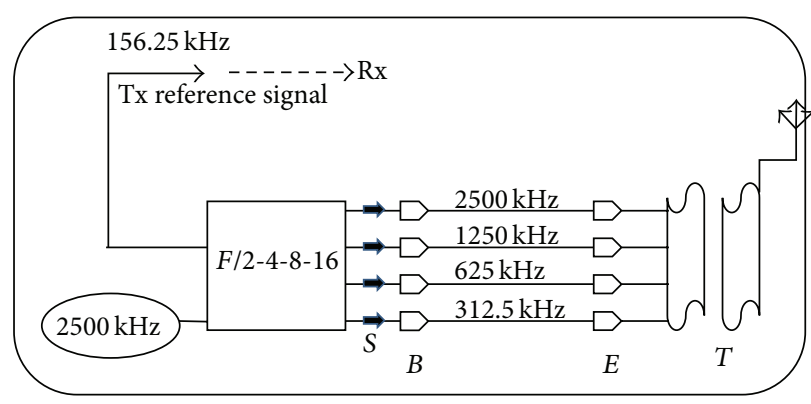

(b)

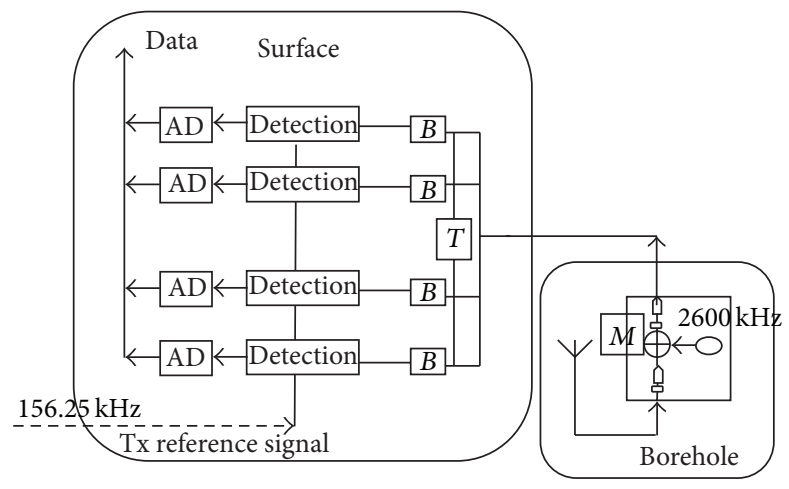

(c)

FIGURE 5: Diagrams of (a) the EMRE system, (b) the transmitter, and (c) the receiver.

TABLE 1: Information on the EMRE system.

\begin{tabular}{lc}
\hline Property & Value \\
\hline Operating frequencies & $312.5,625,1250,2500 \mathrm{kHz}$ \\
Measurement range of voltage & $0.5-1000 \mu \mathrm{V}$ \\
Measurement range of phase & $0-360$ degrees \\
Maximal transmitter power & $2 \mathrm{~W}(33 \mathrm{dBm})$ \\
Diameter of borehole probe & $36 \mathrm{~mm}$ \\
Measurement modes & $\mathrm{ZOM}$ and MOM \\
Winch capacity & $1000 \mathrm{~m}$ \\
Pressure tolerance & $<2000 \mathrm{~m}$ \\
\hline
\end{tabular}

MOM: multioffset measurement, ZOM: zero-offset measurement.

can be a limiting factor. The dynamic range of a receiver is the input power range over which the receiver is useful. Thus, the user has to select the antennas carefully in order to avoid the saturation of the receiver. Changing the antennas, rejecting the problematic frequency, or the usage of longer distances between the transmitter and receiver are ways to eliminate saturation [15]. The technical specifications of the EMRE system are presented in Table 1.

\section{Measurement Technique and System Capacities}

According to the cross-borehole measurement geometry, the EMRE system operates in the transmission mode in order to detect the attenuation of the transmitted radiofrequency waves (Figure 1). The measurements can be performed using MOM (multioffset measurement) or ZOM (zero-offset measurement). MOM is the traditional method, whereby a stable transmitter is fixed in one borehole and a mobile receiver is moved in the other borehole (fan-beam geometry). The main idea is to measure a large number of angles passing through the space between the boreholes. In ZOM, both the transmitter and receiver are moved synchronously in the boreholes (parallel-beam geometry). This is a quick and simple technique to locate and define anomalous attenuation zones. In a homogeneous environment, signal levels should be the same at each location [10].

Two-way measurement is normally performed, where the transmitter and receiver are interchanged in the boreholes, and a full tomographic survey can be accomplished. This is known as a limited angle method, because the angle coverage is seldom more than 60 degrees (cf. medical computerized tomography (CT), which easily has an angle coverage of 360 degrees). When an electromagnetic wave is propagated in a material, three basic mechanisms of multipath waves can be distinguished: reflection, diffraction, and scattering. Multipath and direct waves can combine constructively or destructively depending on their phases in the receiver. The destructive combination of waves can result in a severely attenuated received signal. In addition, radiowaves suffer from strong internal attenuation. The EMRE system cannot distinguish between the above-mentioned phenomena.

According to ray theory (zero wavelength or high frequency approximation), electromagnetic energy propagates along a ray and variations in the electromagnetic field are 


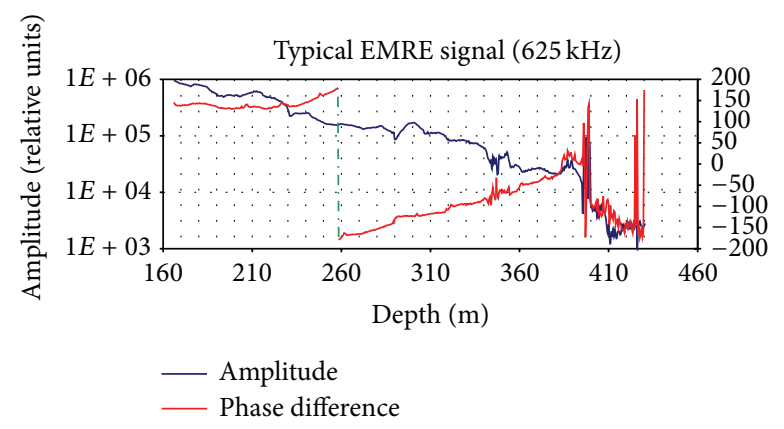

(a)

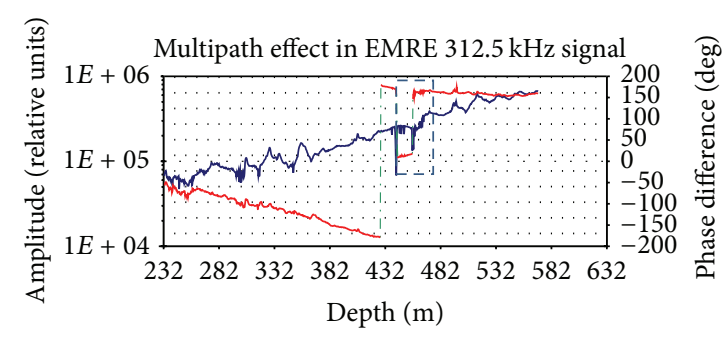

- Amplitude
Phase difference

(b)

Figure 6: (a) A possible multipath effect (Rayleigh scattering) at $442-457 \mathrm{~m}$. The phase has suddenly changed by $\sim 130$ degrees, meaning a longer path of $\sim 2 / 5 \cdot \lambda$ for the multipath wave. The change in the phase at $\sim 427 \mathrm{~m}$ is due to the presentation mode $\left(-180^{\circ}\right.$ to $\left.+180^{\circ}\right)$. Due to the presentation mode, the phase is plotted between $-180^{\circ}$ and $+180^{\circ}$. (b) Recording in a massive sulphide deposit.

generated by changes in the material properties along the ray. In a homogeneous material, the rays are straight lines, unlike in an inhomogeneous material. The volume of the first Fresnel zone (prolate spheroid) is an important factor when considering the rays that have the largest significance in the detection. This volume is a function of wavelength.

The waves that propagate in the first zone (e.g., direct and multipath waves) are generally detectable in the EMRE system. In addition, the smallest structure that can be resolved is estimated by the width of the first Fresnel zone, or objects with dimensions smaller than the width can be distinguished from the background. The width of the Fresnel zone is spatially variable, being the smallest near the transmitter and receiver boreholes and the largest at the midpoint between the boreholes (Table 1). It is an internal property of the EMRE system to always sense the strongest wave (first Fresnel zone), which according to Fermat's principle must also be the shortest in time. When sensing a multipath wave, a sharp and localized change in the measured phase difference immediately tells the operator the difference in the lengths of raypaths, or how much longer a path the multipath wave has travelled. In addition, the phase difference between the direct and reflected wave may interfere in such a manner that the amplitude may increase or decrease. In Figure 6(a), a possible multipath effect is presented at a level of $442-457 \mathrm{~m}$. The phase has changed by 130 degrees.

In Figure 6(b), a typical registration of $625 \mathrm{kHz}$ is presented. The measured amplitudes (not normalized) are plotted in blue and the phases in red. The measurement was carried out in a massive sulphide deposit. The amplitude and phase changes can be very rapid and happen at very short distances, as can be seen in the later parts of the scanning $(390 \mathrm{~m})$. It is evident that the sensitivity of the EMRE system $(\sim 0.5 \mu \mathrm{V}$ is the minimum field strength) is sufficient and detailed amplitude recordings are possible. Thus, the registrations give a valuable insight into the geology of the section and make the planning of measurements easier during scanning.

The performance level of the EMRE system can be estimated using a simplified model [12]. The maximum output power of the EMRE system is $2 \mathrm{~W}(33 \mathrm{dBm})$ and the sensitivity of the receiver (thermal noise level) is $\sim 10^{-13}$ to $10^{-15} \mathrm{~W}$. Thus, defining $b_{\max }=10 \log _{10}\left(2 / 10^{-13}\right) \sim 135 \mathrm{~dB}$ as the ratio of the transmitter power to the power sensitivity of the receiver, the total attenuation losses at the frequency for $b_{\max }$ can be estimated. The results are presented in Table 2 .

As a summary, in the situations where the host rock is highly resistive $(>10000 \Omega \mathrm{m})$, the borehole separation can be up to 1000 metres and the signals are at least detectable at the lowest measurement frequency $(312.5 \mathrm{kHz})$. However, at longer distances, the boreholes must also be deeper so that a reasonable angle coverage is met. It can said that when the transmitter and receiver are lowered deep enough, one would benefit from a gain, referred to as the depth gain, that is similar to the situation where the dipoles are elevated above the ground surface, benefitting from a height gain. At typical borehole separations of 400-600 metres (host rock resistivity $\approx 7500 \Omega \mathrm{m}$ ), the EMRE system can be used across its whole frequency band, despite device-based losses, which have not been taken into account at all. The limited dynamic range of the device sets limits for its use over short distances, where the saturation of the device can make usage impossible.

\section{Processing and Interpretation}

The problem of forming a cross-sectional image or a tomographic reconstruction arises in a variety of contexts, including medical tomography (CT) and radiofrequency imaging (RIM) between two deep boreholes. It is always important to remember that the reconstruction is a registered response of the subsurface medium to the existing electromagnetic fields across the used frequency band. Thus, users must understand the physical meaning of a medium's electrical and magnetic properties and how these relate to the attenuation of electromagnetic fields. General purpose methods consist of real optimization methods where the ultimate goal is to find $x$ that minimizes a cost functional of the form $\|y-A x\|$, where $y$ is a random vector of measurements, $x$ is a vector representing the unknown quantity to be reconstructed, and $A$ is the system matrix. In geotomography, special purpose methods are used. The algebraic reconstruction technique (ART) [26-29] and the simultaneous iterative reconstruction 
TABLE 2: The total attenuation losses $(\mathrm{dB})$ as a function of material parameters and frequency. All other losses (e.g., in the device, different polarizations in transmission, and reception) are excluded.

\begin{tabular}{|c|c|c|c|c|c|c|c|c|c|c|c|c|c|c|}
\hline \multirow{2}{*}{$\begin{array}{l}\rho=2500 \Omega \mathrm{m} \\
\varepsilon_{r}=10 \quad \varepsilon_{r}=4\end{array}$} & \multicolumn{2}{|c|}{$\begin{array}{c}\text { Wavelength } \\
\text { (m) }\end{array}$} & \multicolumn{2}{|c|}{$\begin{array}{c}\text { Skin depths } \\
(\mathrm{m})\end{array}$} & \multicolumn{2}{|c|}{$\begin{array}{l}\text { Attenuation } \\
(\mathrm{dB} / \mathrm{m})\end{array}$} & \multicolumn{2}{|c|}{$\begin{array}{l}\text { Attenuation } \\
\mathrm{dB}-200 \mathrm{~m}\end{array}$} & \multicolumn{2}{|c|}{$\begin{array}{l}\text { Attenuation } \\
\mathrm{dB}-400 \mathrm{~m}\end{array}$} & \multicolumn{2}{|c|}{$\begin{array}{l}\text { Attenuation } \\
\mathrm{dB}-600 \mathrm{~m}\end{array}$} & \multicolumn{2}{|c|}{$\begin{array}{c}\text { Attenuation } \\
\mathrm{dB}-800 \mathrm{~m}\end{array}$} \\
\hline & & & & & & & & & & & & & & \\
\hline $312 \mathrm{kHz}$ & 191 & 141 & 55 & 87 & 0.16 & 0.1 & 100 & 83 & 151 & 117 & 200 & 149 & 248 & 180 \\
\hline $1000 \mathrm{kHz}$ & 85 & 47 & 44 & 84 & 0.19 & 0.1 & 108 & 83 & 168 & 117 & 225 & 149 & 282 & 180 \\
\hline $2500 \mathrm{kHz}$ & 39 & 20 & 42 & 84 & 0.20 & 0.1 & 111 & 83 & 174 & 117 & 234 & 149 & 293 & 180 \\
\hline $5000 \mathrm{kHz}$ & 19 & 9.5 & 42 & 84 & 0.20 & 0.1 & 111 & 83 & 174 & 117 & 234 & 149 & 293 & 180 \\
\hline \multicolumn{15}{|c|}{$\begin{array}{l}\rho=5000 \Omega \mathrm{m} \\
\varepsilon_{r}=10 \quad \varepsilon_{r}=40\end{array}$} \\
\hline $312 \mathrm{kHz}$ & 240 & 149 & 94 & 170 & 0.09 & 0.05 & 81 & 69 & 113 & 89 & 142 & 107 & 171 & 123 \\
\hline $1000 \mathrm{kHz}$ & 92 & 47 & 85 & 168 & 0.1 & 0.05 & 83 & 69 & 117 & 89 & 149 & 107 & 180 & 123 \\
\hline $2500 \mathrm{kHz}$ & 39 & 20 & 84 & 168 & 0.1 & 0.05 & 83 & 69 & 117 & 89 & 149 & 107 & 180 & 123 \\
\hline $5000 \mathrm{kHz}$ & 19 & 9.5 & 84 & 168 & 0.1 & 0.05 & 83 & 69 & 117 & 89 & 149 & 107 & 180 & 123 \\
\hline \multicolumn{15}{|c|}{$\begin{array}{l}\rho=7500 \Omega \mathrm{m} \\
\varepsilon_{r}=10 \quad \varepsilon_{r}=40\end{array}$} \\
\hline $312 \mathrm{kHz}$ & 270 & 150 & 133 & 252 & 0.065 & 0.034 & 73 & 64 & 97 & 80 & 113 & 93 & 140 & 105 \\
\hline $1000 \mathrm{kHz}$ & 93 & 47 & 126 & 252 & 0.068 & 0.034 & 74 & 64 & 99 & 80 & 122 & 93 & 144 & 105 \\
\hline $2500 \mathrm{kHz}$ & 39 & 20 & 126 & 252 & 0.068 & 0.034 & 74 & 64 & 99 & 80 & 122 & 93 & 144 & 105 \\
\hline $5000 \mathrm{kHz}$ & 19 & 9.5 & 126 & 252 & 0.068 & 0.034 & 74 & 64 & 99 & 80 & 122 & 93 & 144 & 105 \\
\hline \multicolumn{15}{|c|}{$\begin{array}{l}\rho=10000 \Omega \mathrm{m} \\
\varepsilon_{r}=10 \quad \varepsilon_{r}=40\end{array}$} \\
\hline $312 \mathrm{kHz}$ & 282 & 151 & 174 & 337 & 0.049 & 0.026 & 68 & 62 & 88 & 75 & 106 & 87 & 122 & 96 \\
\hline $1000 \mathrm{kHz}$ & 91 & 47 & 168 & 336 & 0.051 & 0.026 & 69 & 62 & 89 & 75 & 107 & 87 & 124 & 96 \\
\hline $2500 \mathrm{kHz}$ & 39 & 20 & 168 & 336 & 0.051 & 0.026 & 69 & 62 & 89 & 75 & 107 & 87 & 124 & 96 \\
\hline $5000 \mathrm{kHz}$ & 19 & 9.5 & 168 & 336 & 0.051 & 0.026 & 69 & 62 & 89 & 75 & 107 & 87 & 124 & 96 \\
\hline \multicolumn{15}{|c|}{$\begin{array}{l}\rho=15000 \Omega \mathrm{m} \\
\varepsilon_{r}=10 \quad \varepsilon_{r}=40\end{array}$} \\
\hline $312 \mathrm{kHz}$ & 293 & 151 & 256 & 504 & 0.034 & 0.017 & 64 & 60 & 80 & 71 & 93 & 79 & 105 & 86 \\
\hline $1000 \mathrm{kHz}$ & 94 & 47 & 252 & 504 & 0.034 & 0.017 & 64 & 60 & 80 & 71 & 93 & 79 & 105 & 86 \\
\hline $2500 \mathrm{kHz}$ & 39 & 20 & 252 & 504 & 0.034 & 0.017 & 64 & 60 & 80 & 71 & 93 & 79 & 105 & 86 \\
\hline $5000 \mathrm{kHz}$ & 19 & 9.5 & 251 & 504 & 0.034 & 0.017 & 64 & 60 & 80 & 71 & 94 & 79 & 105 & 86 \\
\hline
\end{tabular}

Bold numbers are referred to as favourable conditions.

technique (SIRT) $[11,30]$ are special algorithms. SIRT and ART are traditional methods that are commonly used even today. ImageWin [31] is a commercial software program for image reconstruction. In addition to the two algebraic reconstruction methods, the iterative least square method (LSQR) [32] and the iterative conjugated gradient method (CGLS) [33] are included. If the electrical contrasts are moderate, the results from the above-mentioned methods are often very good and sufficient. New algorithms that are also effective with limited angle data (data coverage $<180$ degrees) are available in dentistry. They could perhaps also be used in solving complicated geotomographic problems, resulting in more reliable reconstructions of sections.

The data processing is carried out in two stages. In the first stage (editing stage), the raw data are smoothed and sampled. The signals are cleaned to remove the noisy sections, possible multipath effects defined by the short and sharp phase changes (Figure 6(a)), and spurious values (outliers) by rejection and average filtering. Before removing a distinct feature from one frequency, a careful comparison with all frequencies must be performed, and if the feature is missing from the other frequencies, it may be removed [34].

In the second stage, the borehole section is discretized into rectangular cells. The number of raypaths passing through each cell is determined. The densities are the highest in the central parts of the panels. This results in a better and more reliable reconstruction in these regions. On the edges, the density is reduced. Normally, the number of raypaths is important for creating a well-determined tomographic image. However, situations where the raypath lengths increase due to the small angles $\left(<40^{\circ}\right)$ may lead to misleading attenuation distributions. Thus, the raypaths corresponding to small angles should be removed. Due to the measurement geometry the densities are low in the top and bottom parts. 
This may result in artifacts in the reconstruction, or the image may be biased (accumulation of error) towards the areas having fewer raypaths. Thus, the tomographic reconstruction should include a consideration of raypath coverage in order to determine which parts of the model are best constrained by the data.

The amplitude data are interpreted starting from the plane wave assumption, which is valid in the far-field domain. After the discretization of the borehole section into rectangular cells, the measured amplitudes are converted into the attenuation distribution using a data reduction step [11, 35-38]. In the EMRE system, the output power is not monitored, but the unknown source strength can be estimated by a linear regression of reduced amplitudes in a homogeneous medium [39], and the global value is automatically calculated. In a homogeneous environment, a global source strength can be used for all transmitter positions. In situations where close proximity to a borehole wall changes from one transmitter position to another, resulting in large variations in antenna impedance, local source strengths can be used. Because antenna impedance is sensitive to the electrical properties of the material near the borehole, the radiated power can also differ considerably from one transmitter position to another [19]. If the electrical contrasts are moderate, the results from the above-mentioned methods are often very good and sufficient.

Data reduction is completed to provide the attenuation distribution $(\mathrm{dB} / \mathrm{m})$ by using the above-mentioned reconstruction methods. The attenuation distribution can be converted to the conductivity distribution $(\mathrm{S} / \mathrm{m})$ using an equation presented by [39]:

$$
\sigma=\frac{2500 \cdot \alpha^{2}}{\pi^{2} \cdot f},
$$

where $\alpha$ is the attenuation in Nepers/m and $f$ the frequency in $\mathrm{kHz}$. Equation (10) is a plane wave solution that is satisfied in the far-field domain.

The final presentation of the reconstruction is performed using the EMRE toolbox [40]. As a result, the attenuation or conductivity distribution is presented in the $3 \mathrm{D}$ borehole environment. When several sections are included in the measurement plan, it is possible to also delineate the targets that may not be situated directly in the cross section but somewhere in the first Fresnel zone.

\section{Field Case in Finland}

Posiva Oy carries out research and development related to spent nuclear fuel in Finland. The work is concentrated at Olkiluoto in the municipality of Eurajoki (in western Finland). Underground characterization premises, known as ONKALO, have been under construction since 2004. In 2009, a special project was established between Posiva and GTK to determine the capacity and usefulness of RIM in determining the structural integrity of the rock in the area. This was the second time [41] that RIM measurements had been taken in Olkiluoto, because the pioneering work had already been performed in 2005 [21, 22]. The functioning level of the system was incorrect due to the lack of a reference signal; thus, only the amplitude detection was possible. Despite this, the attenuation distributions were in good agreement with the results from other methods used in the area. The massive sulphide deposit at Pyhäsalmi (in central Finland) is an ideal test location for the system, where the resistive host rock is occupied by a massive conductive ore. The test measurements were continued at Pyhäsalmi in 2008 [42].

This paper illustrates the results from Olkiluoto in 2009. The measurements were carried out at Olkiluoto in one borehole section: OL-KR40-OL-KR45 (Figure 7). The holes, drilled with diamond drill-bits, are water-filled and their upper sections lined with steel casing $(40 \mathrm{~m})$. The borehole water may be quite conductive due to dissolved mineral salts. The casing is not a problem in RIM measurements because the starting levels are normally far below the casing depth. The boreholes are $76 \mathrm{~mm}$ in diameter and $\sim 1030 \mathrm{~m}$ in depth. Borehole OL-KR40 is tilted $~ 30$ degrees vertically and the azimuth is $\sim 270$ degrees. Borehole OL-KR45 is tilted $\sim 40$ degrees vertically and the azimuth is $\sim 100$ degrees. The distance between the borehole collars is $\sim 8 \mathrm{~m}$ at the surface, but in deeper parts the distance is $\sim 1050 \mathrm{~m}$. In OLKR40, 88 transmitter locations were tested in the depth range of $100-750 \mathrm{~m}$. In OL-KR10, measurements from 53 transmitters were taken in the depth range of 100-500 m. For each transmitter station, sufficiently long receiver scanning was performed with a dense continuous logging mode (2 readings/s corresponding to $\sim 0.5 \mathrm{~m}$ spacing). The receiver scanning length along the borehole was determined by the tool sensitivity and the signal-to-noise ratio. When the scanning had been completed, the transmitter was moved to the next station. After all transmitter stations in one borehole had been measured, the transmitter and receiver boreholes were interchanged to complete the full tomographic survey. This provides denser and more homogeneous ray coverage in the central parts of the section.

The rocks of Olkiluoto can be divided into two major classes: (1) metamorphic rocks and (2) igneous rocks. The bedrock of Olkiluoto has also been subjected to retrogressive metamorphism, hydrothermal alteration, and surface weathering. The most significant of these features is hydrothermal alteration, resulting in kaolinite and illite clay mineral formation, sulphidisation, and carbonatisation. Brittle deformation is visible as joints, veins, and fissures as well as single plane faults and fault zones. Fractures have three distinct orientation maxima: fractures dipping gently to moderately towards S-SE following the orientation of ductile deformation and two steeply dipping, E-W and N-S striking clusters. These fracture sets form in places more continuous, similarly oriented brittle fault zones. The main fault zones in Olkiluoto gently dip to the southeast [43]. Figure 8, compiled by senior scientist M. Paananen, shows the lithology and brittle fault zones along a vertical section defined by drillholes OL-KR40 and OL-KR45. The section is based on the Geological Model of Olkiluoto Site, Version 2.0 [43]. The geological features are described in the Geological Site Model, in Olkiluoto Site Description [44]. 


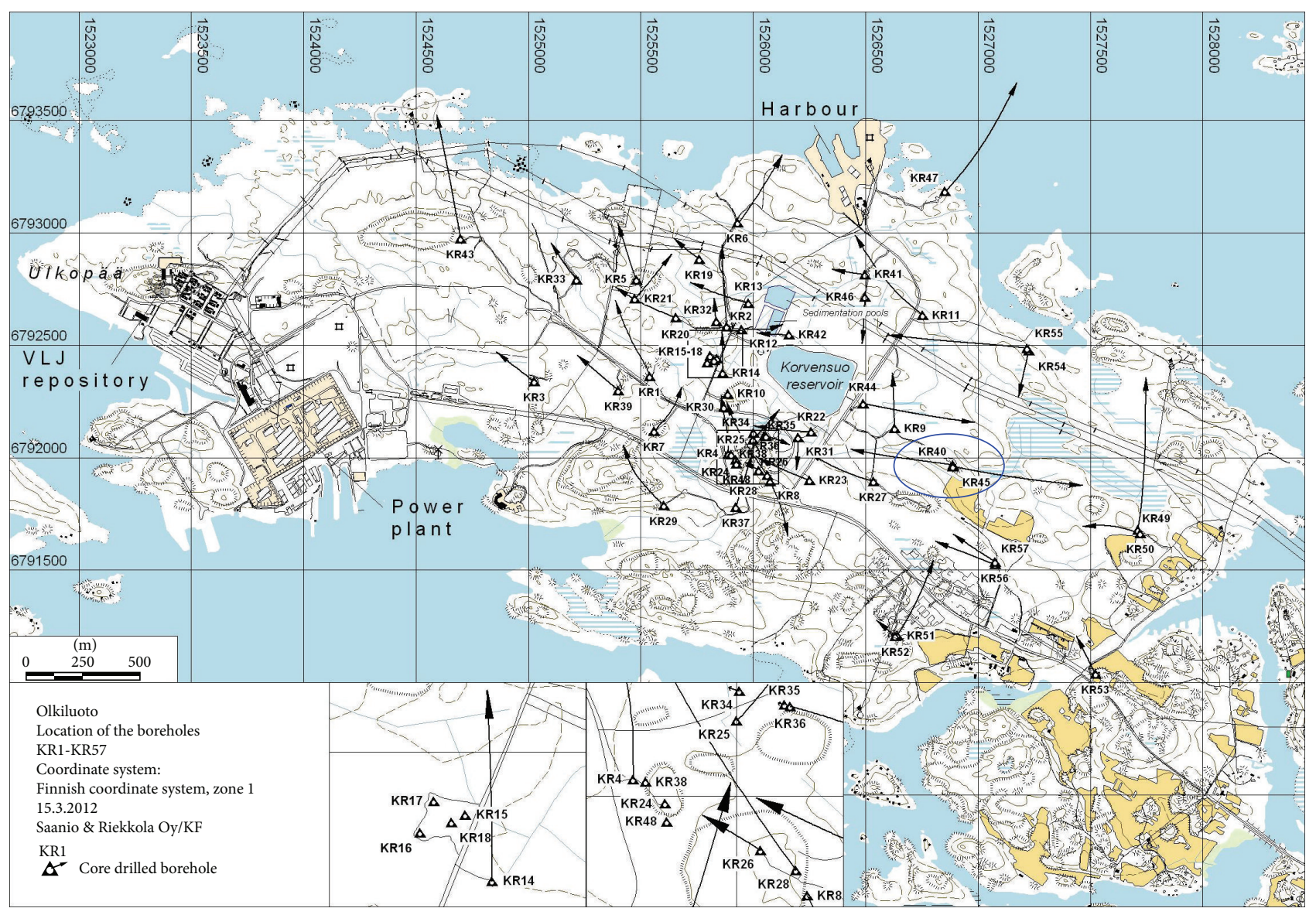

FIgUre 7: The map of Olkiluoto site. The borehole pair (OL-KR40-OL-KR45) is marked by an oval.

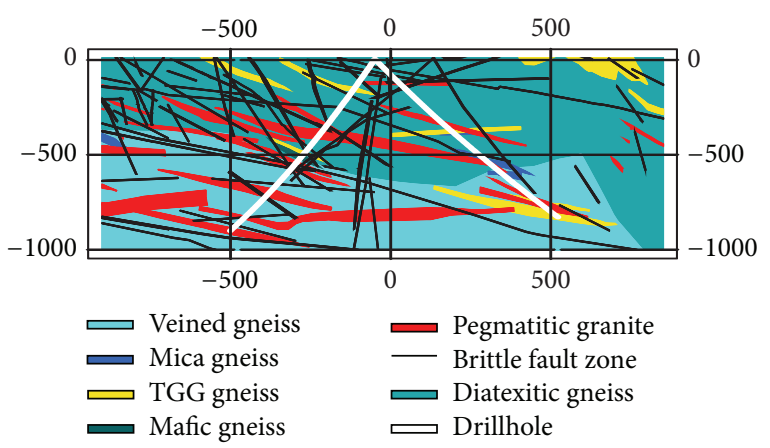

FIGURE 8: Vertical section of drillholes OL-KR40 (white line on the left) and OL-KR45 (white line on the right). Lithology and brittle fault zones. The azimuth of the section is $98^{\circ}$ clockwise from the north. The section is based on the Geological Model of the Olkiluoto Site, Version 2.0 [43].

The borehole section (OL-KR40-OL-KR45) was challenging for the EMRE system because the geometry was conic. The mutual orientation of the antennas was occasionally very disadvantageous and generated problems. On the other hand, the shape of the section also made it possible to start the measurements closer to the Earth's surface without one dominant wavelength rule to avoid the possible reflection from the Earth's surface. Alternatively, the scanning could be started near the Earth's surface when the transmitter was located deeper. The highest measurement frequency $(2500 \mathrm{kHz})$ was problematic, as no signals were received. The reason lay not only in the internal attenuation of the material but also in the unfavourable functioning of the transmitter antenna [19]. The theoretical scattering parameter $\mathrm{s}_{11}$, which defines the relationship between the input power and the reflected power in the transmitter, was $\sim-2 \mathrm{~dB}$, thus meaning that $\sim 62 \%$ of the power was reflected back to the generator and only $\sim 38 \%$ was radiated. In addition, the large polarization losses in antennas can also have been significant due to the problematic orientation of the boreholes.

The interpretation was performed by using ImageWin software. The final results with two lower frequencies are presented in Figures 9(a)-9(b). As a whole, the results coincide very well. The maximum attenuation rates range from $0.77 \mathrm{~dB} / \mathrm{m}(625 \mathrm{kHz})$ to $0.7 \mathrm{~dB} / \mathrm{m}(312.5 \mathrm{kHz})$. The corresponding apparent resistivity value is $\sim 200 \Omega \mathrm{m}$ at both frequencies (Figure 3 ). The main and common attenuating zones are clearly visible in the images.

The short normal curves in Figures 8(a)-8(b) illustrate the distribution of electrical properties of the bedrock in drillholes OL-KR40 [45] and OL-KR45 [46]. Within the uppermost $600 \mathrm{~m}$ in OL-KR40, variation in resistivity is 


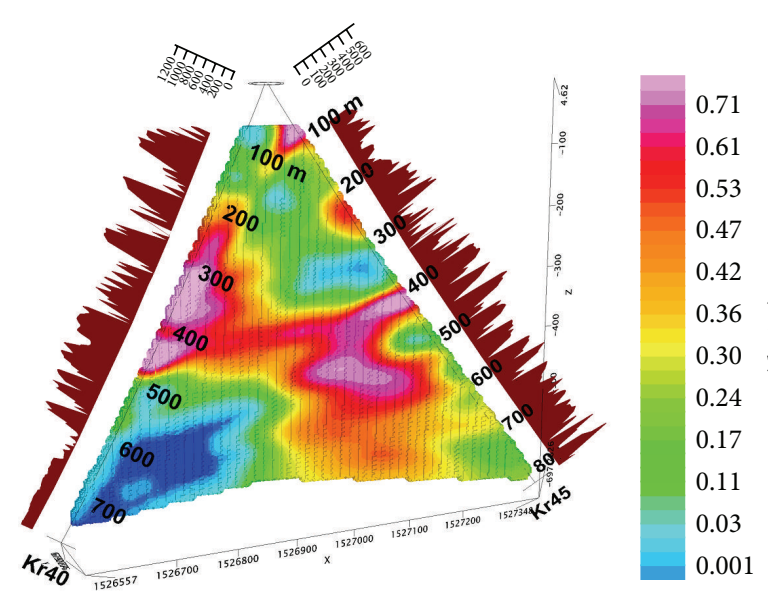

(a)

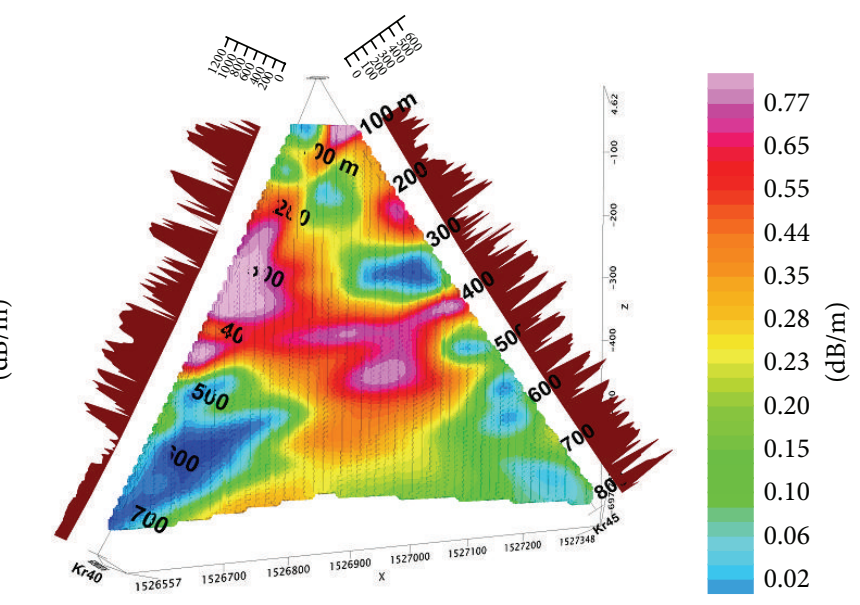

(b)

Figure 9: The attenuation distribution of section OL-KR40-OL-KR45: (a) $312.5 \mathrm{kHz}$, (b) $625 \mathrm{kHz}$. Short normal curves ( $\Omega \mathrm{m}$ ) are added to the images and plotted as brown filled profiles.

rather high, the base level being $\sim 1000 \Omega \mathrm{m}$. There are several distinct resistivity minima, indicating resistivity values of $10 \Omega \mathrm{m}$ or even less. These minima are undoubtedly caused by mineral conductors, mainly sulphide or graphite. Most of them can also be correlated with deformation zones, carrying groundwater, sulphides, and occasional graphite. In drillhole OL-KR45, the variation is less distinct, usually limited between 100 and $10000 \Omega \mathrm{m}$. According to geological logs $[47,48]$, the bedrock around OL-KR45 is generally more intact compared to OL-KR40; some deformation zones are intersected by OL-KR45 within the first $200 \mathrm{~m}$, but at deeper levels the fracturing is mostly slight. It is probable that the several minima observed in the short normal curve of OL-KR45 are related to sulphide that has resulted from pervasive or fracture-controlled hydrothermal alteration of the bedrock.

The radio imaging results between deep drillholes OLKR40 and OL-KR45 indicate variation in the attenuation of amplitude. Variation in the attenuation of the electromagnetic field is associated with variation in the bulk electrical conductivity of the rock mass. Migmatitic and pegmatitic rocks in Olkiluoto generally display very low conductivity, which enables good penetration of radiowaves, and low attenuation.

Three main reasons exist for increased attenuation:

(1) elevated porosity of some rock volumes, possibly associated with fractures or alteration and combined with an increased content of highly saline bedrock groundwater;

(2) sulphide alteration, mainly producing pyrrhotite and pyrite in the rock mass or on fracture coating, sometimes also associated with graphite occurrences;

(3) brittle deformation zones, containing a higher water content due to fracturing, clay mineral occurrences, and sulphide and graphite occurrences.
Radio imaging results can be assessed along with drillhole logging data and the geological model of the site. The upper parts of both OL-KR40 to the depth of $530 \mathrm{~m}$ and OL-KR45 to the depth of $620 \mathrm{~m}$ are dominated by diatexitic gneiss. Short normal resistivity logging displays the varying occurrences of conductive locations. Many of these are associated with sulphides, according to the elevated susceptibility between $100-400 \mathrm{~m}$ in OL-KR40 and $0-300 \mathrm{~m}$ in OL-KR45. These locations coincide fairly well with elevated attenuation near the drillholes in RIM results. Below the borehole depth of $530-620 \mathrm{~m}$, the dominant rock type is veined gneiss, with much less conductive occurrences, which is also illustrated by lower attenuation in the RIM image. However, the salinity of the groundwater is greatly elevated below these depths. The RIM image displays the boundaries of the rock mass having elevated attenuation. The volume of higher attenuation near OL-KR40 in the drillhole depth range of 200-450 m appears to continue eastward with a gentle apparent dip. This may be associated with the typical orientation of ductile deformation and the brittle fault zones. The feature may not continue with the same orientation to OL-KR45, which is supported by the geological model, according to which not all of the major brittle deformation zones continue between the drillholes. Hence, there may be a discontinuity or termination of geological structures between these drillholes. RIM data do not precisely display the form of the zones, but rather they show the rock volume having elevated mineral occurrences. Other geophysical methods, such as seismics, may indicate the actual form of these objects.

\section{Conclusions}

The EMRE system has been taken into productive use at the Geological Survey of Finland (GTK) following a set of test measurements at Olkiluoto [22, 41] and Pyhäsalmi [42]. The system is completed by a graphical interface, EMRE GUI [34], in which the preliminary editing stage is performed, and 
by a commercial interpretation program (ImageWin) for the tomographic reconstruction. The final presentation is carried out with the EMRE module (implemented in Geosoft), in which the attenuation or conductivity distribution is transferred and displayed in the 3D borehole environment [40]. The three different cases have proven the potential of the method in exploration geophysics.

The transmitter is a continuous wave (CW) device and all the measurement frequencies are sent simultaneously in one output signal. The nominal output power is $2 \mathrm{~W}(33 \mathrm{dBm})$. Impedance matching provides the means to maximize the power transfer or minimize the reflections from the load (antenna). Whenever a transmitter generator operates with a load, the maximum possible power is delivered to the load when the impedance of the load is equal to the complex conjugate of the impedance of the source. For two impedances to be complex conjugates, their resistances must be equal, and their reactances must be equal in magnitude but of opposite signs. The matching problem has not been solved in our transmitter due to the broad simultaneous frequency band and highly variable properties of the environment, which make the solution impossible. Thus, the total power radiated from the antenna into the rock is reduced. On the other hand, halving the power only means a decrease of $3 \mathrm{dBm}$. In the sections (e.g., closely located boreholes, saturation) where the response of a frequency or frequencies may be fuzzy, the rejection of a frequency or frequencies from the normal operation can be performed in a jumper board. Thus, the total power is channelled to the remaining frequencies. The receiver is a superheterodyne-type receiver and it deviates little from a normal radio receiver. The reception and the intermediate frequency (IF) synthesis are performed down in the borehole receiver. The IFs are used as carriers to feed the measured signals through the winch cable up to the Earth's surface where the final detection is performed. The same mismatching problem concerns the receiver, but it is not as crucial as in the transmitter [15]. The dynamic range of a receiver is the input power range over which the receiver is useful. The dynamic range of the EMRE receiver is $\sim 40 \mathrm{~dB}$. In principle, the EMRE receiver consists of two receivers: one is in the borehole and the other at the surface (Figure 5). The technical specifications of the RIM system are presented in Table 1. Figure 1 presents the main parts of the measurement system.

The idea of EMRE device is excellent. Although the electronic construction is complicated, the device is a work of art. In fieldwork, two operators are needed, one in the transmitter and another in the receiver station, and the measurement installation can be quickly performed. A crossborehole EM survey has several clear benefits over groundlevel electromagnetic sounding methods. Applying a borehole source, it brings the survey closer to the target and allows the use of higher frequencies, thus enabling a higher spatial resolution. Another benefit is the possibility to view the target from different angles and directions, not only in the vertical direction. Having the source in a borehole eliminates the boundary effects related to the ground surface and the strong attenuation emerging from soil deposits. A drawback is the suboptimal availability and location of boreholes. The amplitudes of the electric field along the receiver borehole axis and the relative phase difference are measured (Figure 1). A reference signal is generated in the transmitter borehole unit and fed through cables to the receiver unit at the Earth's surface. This is an essential signal when tuning the receiver. Special filters are used to galvanically isolate the antennas from the winch cables (technique used in the trap dipole). Measurements are controlled and information is recorded with a portable computer. Scanning speeds (receiver movement) can be as high as $30 \mathrm{~m} / \mathrm{min}$ without any disturbance. The automated measurement process requires minimal intervention by the operator. The operator can monitor the data quality at all the frequencies in real time or view previous results. This allows adjustments to the survey depth range and sampling density and will enable decisions on remeasurement (Figure 1). The device is rather rapid to be applied and the technique is feasible for electromagnetic characterization. The spatial resolution is better, the application is faster than with the cross-borehole resistivity imaging, and the penetration range of investigation is greater than the 30$50 \mathrm{~m}$ obtained with a $20 \mathrm{MHz}$ cross-borehole radar (which is also slower to be applied). Conversely, a cross-borehole radar would provide velocity data controlled by the dielectric permittivity, associated with the water content and lithology, whereas a trade-off of RIM when compared to radar is that arrival time measurement for the dielectric permittivity (relevant for lithology mapping) is not available. The relative phase difference data of the EMRE system contain information on the slowness, which has been applied in the estimation of dielectric permittivity $[24,25]$. Dense receiver stations allow the compilation of reasonably smooth tomographic images. Some requirements for successful borehole surveys can be mentioned. The section should be roughly in a common plane, with a minimum starting depth of 100-200 m below the ground surface, and the boreholes should be deeper than the distance between them. It could also be possible to start the scanning at even shallower depths when the transmitter is located in deeper parts of the boreholes. A scanning aperture of 30-50 degrees is necessary to achieve a good tomographic reconstruction. The previous electromagnetic and electrical sounding interpretations from the ground surface and boreholes cannot reach the detailed level of the RIM. The interpretation used is based on the far-field solution of an electric field. The straight-ray assumption is accepted, the electromagnetic energy thus propagating along a ray, and changes in the electromagnetic field are generated by changes in the material properties along the ray. Curved raypaths should generally not be used in amplitude inversion, because the raypath curvature is determined by the velocity, not the attenuation. However, in a low- $Q(Q \ll$ 0.1 ) regime, the slowness (1/velocity) is proportional to the attenuation, and curved raypaths could be used [11]. However, when the contrasting electrical properties are moderate, the simple assumption works well. The full-wave field solution of Maxwell's equations for the electrical dipole field would be useful in complicated and inhomogeneous situations, but no software is available that could take into account the complicated and more realistic characteristics of the environment and the details of borehole antennas and solve 
the problem in a reasonable computer time. Practicable solutions to develop the interpretation could be found in dentistry, where the limited angle problem has been resolved in the angle coverage of $<180^{\circ}$ (limited angle tomography).

The weakness of present device is in its construction. The core of the system is an unstabilized crystal oscillator. It determines the accuracy of the whole system, and because the oscillator is common-collector connected without any adjustments or compensations, it is very sensitive to changes in source voltage and temperature. The limited dynamic range of the device $(\sim 40 \mathrm{~dB})$ sets limits for its use over short distances, where the saturation of the device can make the usage impossible. It should be at the level of $60 \mathrm{~dB}$, which could easily be reached by a modern receiver. The source term estimation (regression based) also affects the results. The resolution decreases as a function of decreasing frequency, and the images thus become more smeared when the frequency is reduced. The smearing effect in the horizontal direction is always a common feature of the reconstructions, despite the frequency used, due to the low angle coverage in that direction. On the contrary, the vertical direction does not suffer from smearing. As the amplitude is the directly measurable parameter, it can be used to estimate attenuation, bearing in mind the following. The far-field condition is assumed where the source and transmitter are separated by several wavelengths. This may not be completely true in all EMRE surveys, but the approximation makes the interpretation much easier. The attenuation $\alpha$ is formulated for the $E_{\theta}$ component, having $1 / r$ dependency in the intermediate and far-field approximation. In the near field, the radial $E_{r}$ component with a different attenuation rate and the static field behaviour, having a dependency of $r^{-3}$ with distances of less than 50-100 m, should be considered. Offplane conductive targets will also strongly influence the field results. Thus, numerical inversion would resolve the case in more detail than approximate and rapid methods such as the straight-ray method.

This paper describes the use of the RIM to determine the apparent resistivity associated with the structural integrity of the rock in the potential area of spent nuclear fuel repository. This was the second time that the EMRE system had been utilized in the region [21, 49]. The final conclusion is that the radiowave imaging method (RIM) appeared well suited to the production of subsurface electrical resistivity properties and continuity information between boreholes in Olkiluoto. The theoretical investigations also support these assumptions; as in situations where the host rock is highly resistive ( $>10000 \Omega \mathrm{m}$ ), the borehole separation can be up to 1000 metres and the signals are at least detectable at the lowest measurement frequency $(312.5 \mathrm{kHz})$. The wavelengths from tens to hundreds of metres in the frequency band (312.5$2500 \mathrm{kHz}$ ) dictate the resolution or the smallest features that can be resolved in the bedrock. Higher frequencies (shorter wavelengths) allow smaller features to be resolved, but at the same time the ranges are shorter because the energy transmitted is strongly attenuated. Lower frequencies (longer wavelengths) have less resolving power but a greater range. Thus, there is a trade-off between the physical resolution and the range of a survey. The smallest detectable objects need to be 20-30 m thick and continuous in the lengthwise direction. The thickness corresponds to the wavelength of the highest measurement frequency $(2500 \mathrm{kHz})$ in a moderately conductive environment. The sulphide-bearing zone below $100 \Omega \mathrm{m}$ contrasts with the high, over $10000 \Omega \mathrm{m}$ granite, and the gneiss mass is adequate to produce a good response with the method. As the conductive bodies are not ideally planar and continuous between boreholes and because the used frequency range will also provide information from volumes outside of the vertical image plane, it is necessary to combine the data with available background information. This work also serves as a good feasibility study of the technique. The method may be further applied in the Olkiluoto area, between other suitable borehole pairs. The RIM was proven feasible in the crystalline rock investigation when compared to the other available electrical methods. The method can provide continuity information between borehole intersections. In sulphide layer detection, it provides similar but more detailed information compared to the Mise à la masse method [49]. In resistive layer detection and cross-borehole geometry, it is probably the only available reliable technique with a slower electrical resistivity tomography (ERT).

\section{Conflict of Interests}

The author declares that there is no conflict of interests regarding the publication of this paper.

\section{Acknowledgments}

The author expresses his appreciation to Posiva Oy for permission to present the RIM and other appropriate geophysical data. He especially wish to acknowledge RF specialist Mika Niemelä for the maintenance of the EMRE device. He would like to thank geophysicist Eero Heikkinen (Pöyry $L t d)$ and senior scientist Markku Paananen (GTK) for their contribution to clarify the geological structure of the borehole section under investigation and comparisons with other geophysical data. Geophysicists Tapio Ruotoistenmäki and Kimmo Korhonen aided in the preparation of this paper. Personal discussions with Dr. Declan Vogt have been very rewarding.

\section{References}

[1] J. R. Wait, "Electromagnetic fields of sources in lossy earth," in Antenna Theory, R. E. Collin and F. J. Zucker, Eds., chapter 23, McGraw-Hill, New York, NY, USA, 1969.

[2] D. L. Lager and R. J. Lytle, "Determining a subsurface electromagnetic profile from high-frequency measurements by applying reconstruction-technique algorithms," Radio Science, vol. 12, no. 2, pp. 249-260, 1977.

[3] R. J. Lytle, E. F. Laine, D. L. Lager, and D. T. Davis, "Using cross borehole electromagnetic probing to locate high contrast anomalies," Geophysics, vol. 44, no. 10, pp. 1667-1676, 1979.

[4] S. F. Somerstein, M. Berg, D. Chang et al., "Radio-frequency geotomography for remotely probing the interiors of operating mini- and commercial sized oil-shale retorts," Geophysics, vol. 49 , no. 8 , pp. $1288-1300,1984$. 
[5] L. G. Stolarczyk and R. C. Fry, "Radio imaging method (RIM) or diagnostic imaging of anomalous geologic structures in coal seam waveguides," Transactions of Society for Mining, Metallurgy and Exploration, vol. 288, pp. 1806-1814, 1986.

[6] G. Buselli, "Electrical geophysics in the USSR," Geophysics, vol. 45, no. 10, pp. 1551-1562, 1980.

[7] D. Vogt, The modelling and design of radio tomography antennas [Ph.D. thesis], University of York, York, UK, 2000, http://www.opengrey.eu/item/display/10068/624370.

[8] J. Cao, Z. He, J. Zhu, and P. K. Fullagar, "Conductivity tomography at two frequencies," Geophysics, vol. 68, no. 2, pp. 516-522, 2003.

[9] K. D. Mahrer, "Review of the radio frequency (RIM) method and its utilization in near-surface investigations," The Leading Edge, vol. 14, no. 4, pp. 249-256, 1995.

[10] H. M. Jol, Ground Penetrating Radar: Theory and Applications, Elsevier Science, Amsterdam, The Netherlands, 1st edition, 2009.

[11] M. J. Jackson and D. R. Tweeton, 3DTOM-Three-Dimensional Geophysical Tomography, Report of Investigation no. 9617, USBM, 1996.

[12] A. Prałat and R. Zdunek, "Electromagnetic geotomographyselection of measuring frequency," IEEE Sensors Journal, vol. 5, no. 2, pp. 242-250, 2005.

[13] J. T. deBettencourt and R. A. Surcliffe, "Studies in deep strata radio communication," Final Report, Raytheon Company, 1962.

[14] P. K. Fullagar, D. W. Livelybrooks, P. Zhang, A. J. Calvert, and Y. $\mathrm{Wu}$, "Radio tomography and borehole radar delineation of the McConnell nickel sulfide deposit, Sudbury, Ontario, Canada," Geophysics, vol. 65, no. 6, pp. 1920-1930, 2000.

[15] A. Korpisalo, "Geotomographic studies for ore explorations with the EMRE system," Measurement, vol. 48, pp. 232-247, 2014.

[16] J. A. Stratton, Electromagnetic Theory, McGraw-Hill, New York, NY, USA, 1941.

[17] K. Zhang and D. Li, Electromagnetic Theory of Microwaves and Optoelectronics, Springer, 2007.

[18] P. T. Lafleche, Underground UHF-EM transillumination: a feasibility study [Ph.D. thesis], The Department of Geological Sciences, McGill University, Montreal, Canada, 1985.

[19] A. Korpisalo, "Borehole antenna considerations in the EMRE system: frequency band $312.5-2500 \mathrm{kHz}$," The Open Geology Journal, pp. 63-79, 2013.

[20] D. J. Daniels, Surface Penetrating Radar, The Institution of Electrical Engineers, London, UK, 1996.

[21] A. Korpisalo, T. Jokinen, N. Popov et al., "Review of crosshole radiowave imaging (FARA) in borehole sections OL-KR4-OLKR10 and OLKR10-OL-KR2 in Olkiluoto," Posiva Working Report 2008-79, 2005.

[22] E. Heikkinen, A. Korpisalo, T. Jokinen et al., "Crosshole radiowave imaging (RIM) at Eurajoki Olkiluoto, Finland," in Proceedings of the 12th European Meeting of Environmental and Engineering Geophysics, Helsinki, Finland, 2006.

[23] A. Korpisalo, "FARA -laitteen kokoonpano ja käyttö," Archive Report Q15/2007/13, Geological Survey of Finland, 2007 (Finnish).

[24] G. V. Redko, L. V. Lebedkin, A. Shuval-Sergeev, K. Stevens, and G. Kazda, "Borehole electromagnetic geophysical methods in ore and coal deposits," in Proceedings of the International Conference "300 Years of Russian Mine-Geological Service", Saint-Petersburg, Russia, 2000.
[25] K. Stevens, A. Watts, and G. Redko, "In-mine applications of the radio-wave method in the Sudbury igneous complex," in Proceedings of the International Conference on Mine Geophysics, Saint-Petersburg, Russia, 1998.

[26] A. C. Kak and M. Slaney, Principles of Computerized Tomographic Imaging, IEEE Press, 1998.

[27] S. Kaczmarz, "Angenaherte Auflosung von Systemem linearer Gleichungen," Bulletin de l'Academie Polonaise des Sciences et Lettres A, vol. 35, pp. 355-357, 1937.

[28] K. Tanabe, "Projection method for solving a singular system of linear equations and its applications," Numerische Mathematik, vol. 17, no. 3, pp. 203-214, 1971.

[29] C. Popa and R. Zdunek, "Kaczmarz extended algorithm for tomographic image reconstruction from limited-data," Mathematics and Computers in Simulation, vol. 65, no. 6, pp. 579-598, 2004.

[30] A. V. Lakshminarayanan and A. Lent, "Methods of least squares and SIRT in reconstruction," Journal of Theoretical Biology, vol. 76, no. 3, pp. 267-295, 1979.

[31] P. K. Fullagar, ImageWin Geophysical Tomography, Fullagar Geophysics Pty Ltd, Queensland, Australia, 2004.

[32] C. Paige and M. Saunders, "LSQR: an algorithm for sparse linear equations and sparse least squares," ACM Transactions on Mathematical Software, vol. 8, pp. 43-71, 1982.

[33] P. C. Hansen, Rank-Deficient and Discrete Ill-Posed Problems, SIAM, Philadelphia, Pa, USA, 1998.

[34] A. Korpisalo, "Graafinen käyttöliittymä FARA -datan käsittelyyn ja tulkintaan,” Archive Report Q16.2/2006/3, Geological Survey of Finland, 2007 (Finnish).

[35] K. Holliger, M. Musil, and H. R. Maurer, "Ray-based amplitude tomography for crosshole georadar data: a numerical assessment," Journal of Applied Geophysics, vol. 47, no. 3-4, pp. 285298, 2001.

[36] K. Holliger and T. Bergmann, "Numerical modeling of borehole georadar data," Geophysics, vol. 67, no. 4, pp. 1249-1257, 2002.

[37] B. Zhou and P. K. Fullagar, "Delineation of sulphide ore-zones by borehole radar tomography at Hellyer Mine, Australia," Journal of Applied Geophysics, vol. 47, no. 3-4, pp. 261-269, 2001.

[38] G. A. Pears and P. K. Fullagar, "Weighted tomographic imaging of radio frequency data," Exploration Geophysics, vol. 29, pp. 554-559, 1998.

[39] B. Zhou, P. K. Fullagar, and G. N. Fallon, "Radio frequency tomography trial at Mt Isa Mine," Exploration Geophysics, vol. 29, pp. 675-679, 1998.

[40] A. Korpisalo, "Radioluotausmittaus (EMRE) -tulosten esittäminen OasisMontaj:ssa," Archive Report Q16.2/2009/14, Geological Survey of Finland, 2008 (Finnish).

[41] A. Korpisalo and M. Niemelä, "Cross-borehole research with EMRE-system: radiofrequency Measurements in drillhole section OL-KR40-OL-KR45 at Olkiluoto," Posiva Working Report 2010-24, 2009.

[42] A. Korpisalo, RIM measurements in three borehole sections in the Pyhäsalmi area with the EMRE system [Ph.D. thesis], unpublished, 2013.

[43] I. Aaltonen, M. Lahti, J. Engströn et al., "Geological model of the Olkiluoto site, version 2.0," Working Report 2010-70, Posiva Oy, Eurajoki, Finland, 2010.

[44] Posiva Oy, Olkiluoto Site Description 2011. Posiva Report 2011-02, 2011. 
[45] A. M. Tarvainen, "Geophysical drillhole logging of the drillholes OL-KR40, OL-KR41, OL-KR41B, OL-KR42, OL-KR42B, OLKR43 and OL-KR43B at Olkiluoto 2006 and 2007," Working Report 2007-38, Posiva Oy, Eurajoki, Finland, 2007.

[46] A. M. Tarvainen and E. Heikkinen, "Geophysical drillhole logging of the drillholes OL-KR43, OL-KR44, OL-KR44B, OLKR45, OL-KR45B, OL-KR46 and OL-KR46B, OL-KR47, OLKR47B and OL-KR48 at Olkiluoto 2007 and 2008," Working Report 2008-61, Posiva Oy, Eurajoki, Finland, 2008.

[47] V. Pussinen and R. Niinimäki, "Core drilling of deep drillhole OL-KR40 at Olkiluoto in Eurajoki 2005-2006," Working Report 2006-49, Posiva Oy, Eurajoki, Finland, 2006.

[48] V. Toropainen, "Core drilling of deep drillhole OL-KR45 at Olkiluoto in Eurajoki 2007,” Working Report 2007-95, Posiva Oy, Eurajoki, Finland, 2007.

[49] A. Korpisalo and E. Heikkinen, [Ph.D. thesis], 2014. 

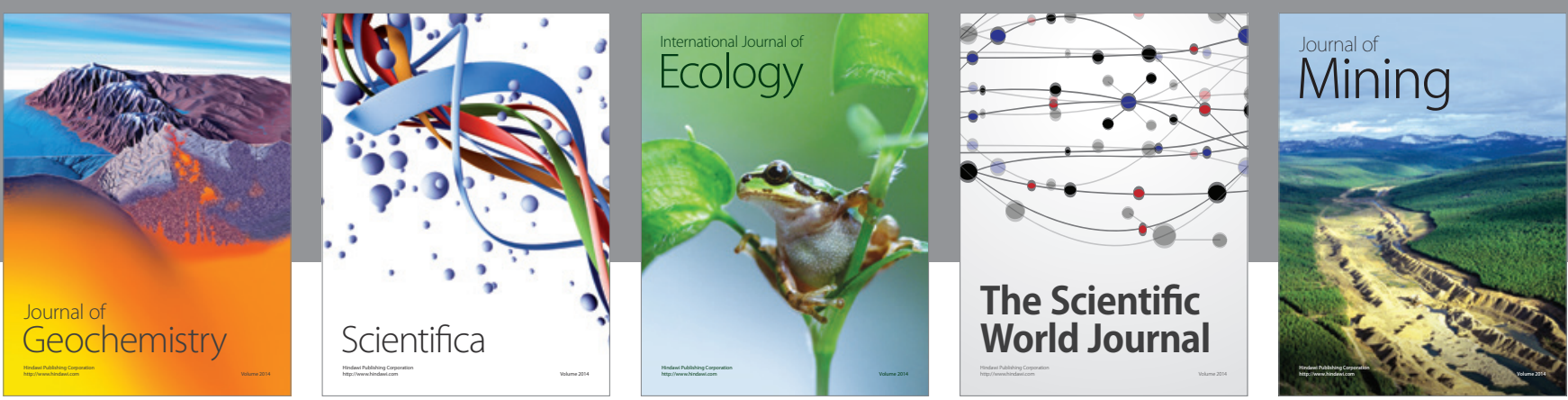

The Scientific World Journal
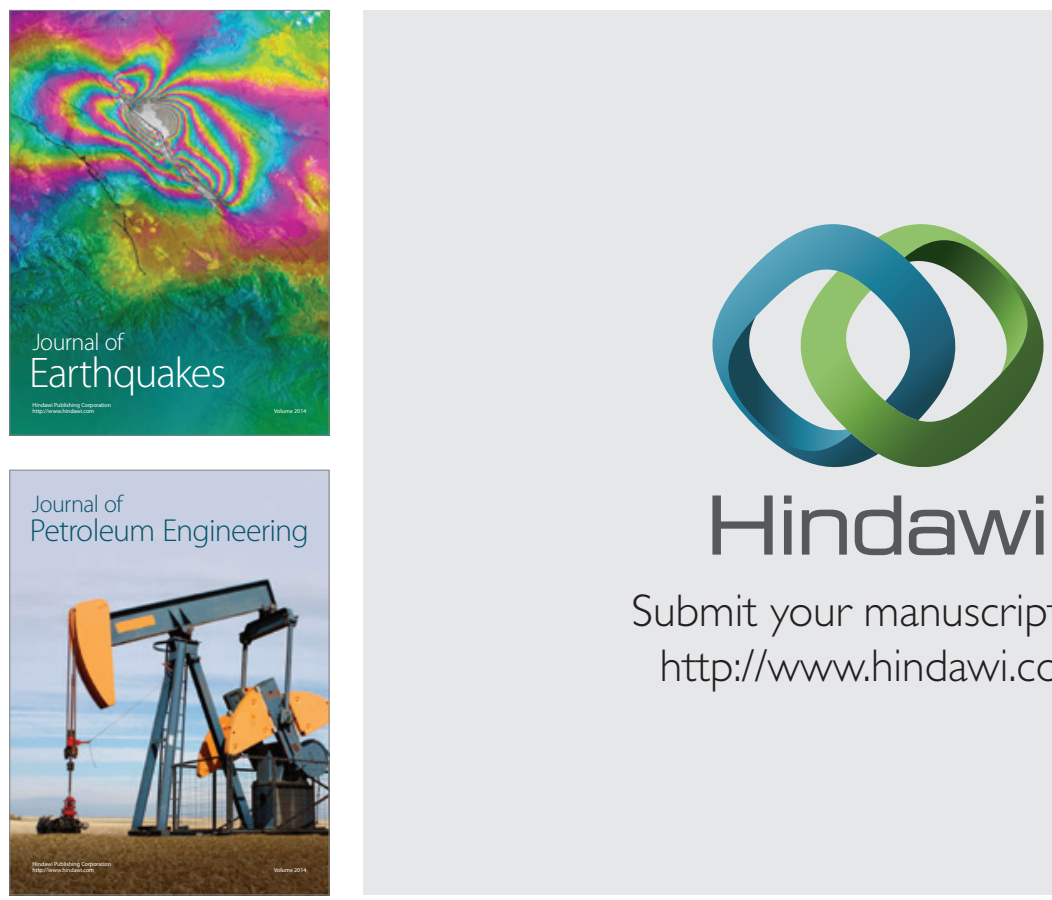

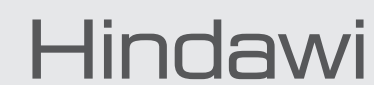

Submit your manuscripts at

http://www.hindawi.com
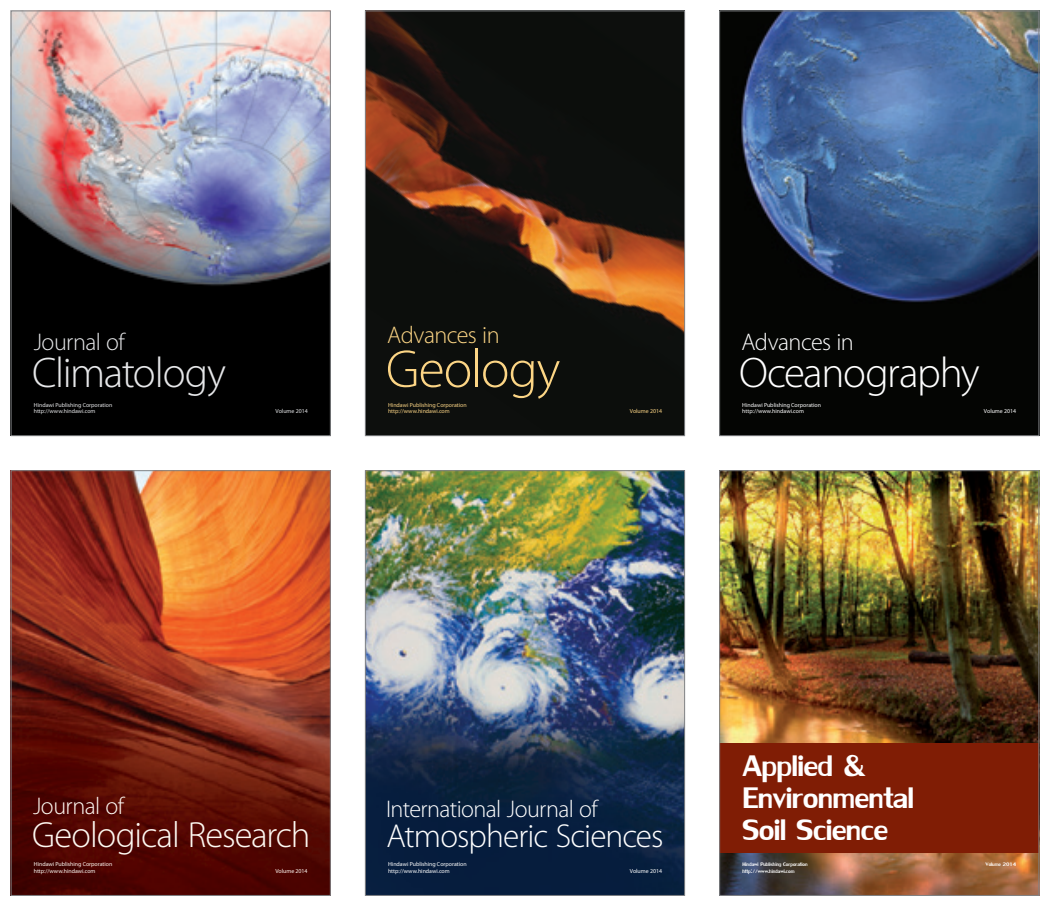
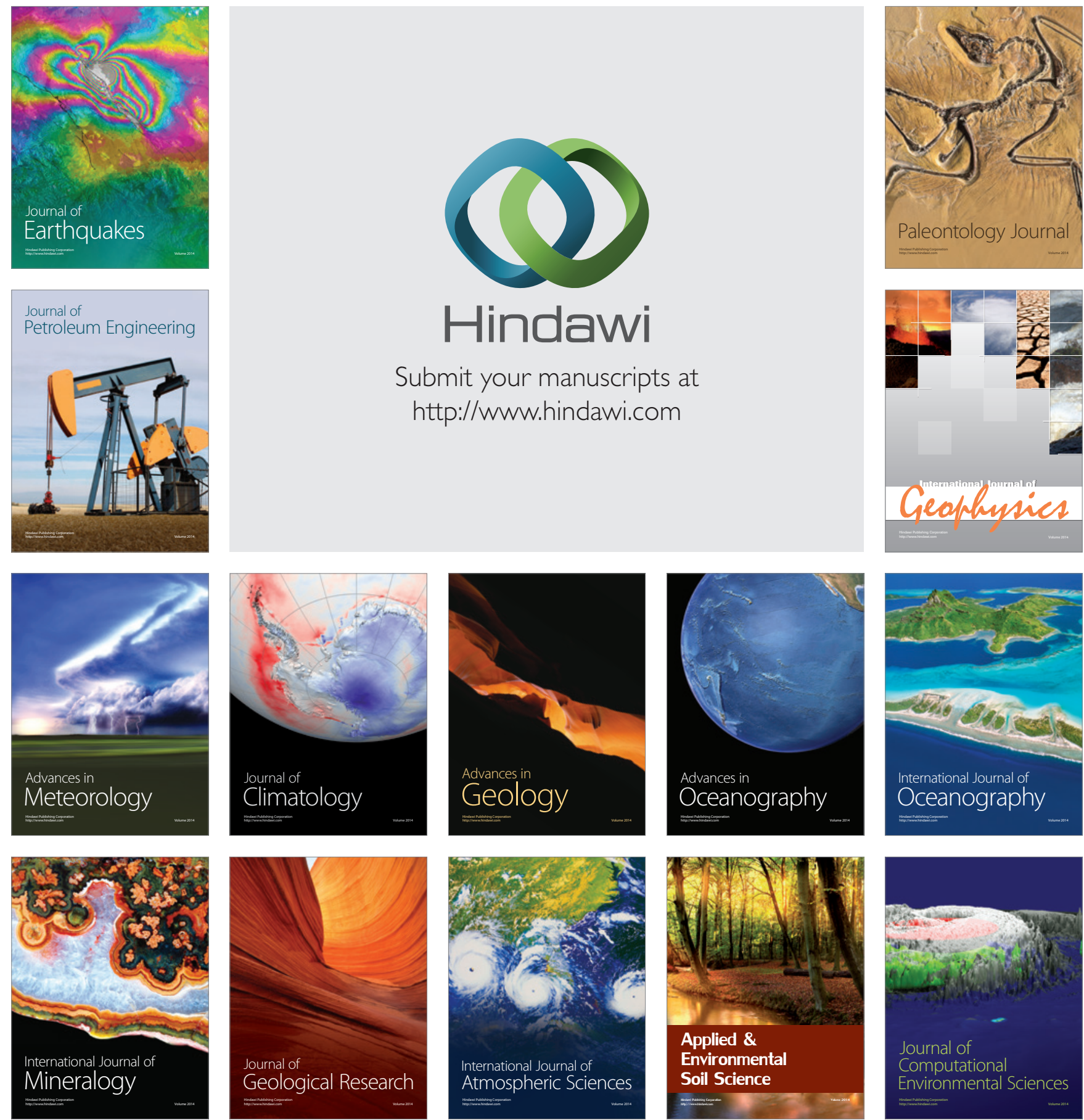